\title{
Flavor universal resonances and warped gravity
}

\author{
Kaustubh Agashe, Peizhi Du, Sungwoo Hong and Raman Sundrum \\ Maryland Center for Fundamental Physics, Department of Physics, \\ University of Maryland, College Park, MD 20742, U.S.A. \\ E-mail: kagashe@umd.edu, pdu@umd.edu, sungwoo83hong@gmail.com, \\ raman@umd.edu
}

ABSTRACT: Warped higher-dimensional compactifications with "bulk" standard model, or their AdS/CFT dual as the purely 4D scenario of Higgs compositeness and partial compositeness, offer an elegant approach to resolving the electroweak hierarchy problem as well as the origins of flavor structure. However, low-energy electroweak/flavor/CP constraints and the absence of non-standard physics at LHC Run 1 suggest that a "little hierarchy problem" remains, and that the new physics underlying naturalness may lie out of LHC reach. Assuming this to be the case, we show that there is a simple and natural extension of the minimal warped model in the Randall-Sundrum framework, in which matter, gauge and gravitational fields propagate modestly different degrees into the IR of the warped dimension, resulting in rich and striking consequences for the LHC (and beyond). The LHC-accessible part of the new physics is AdS/CFT dual to the mechanism of "vectorlike confinement", with $\mathrm{TeV}$-scale Kaluza-Klein excitations of the gauge and gravitational fields dual to spin-0,1,2 composites. Unlike the minimal warped model, these low-lying excitations have predominantly flavor-blind and flavor/CP-safe interactions with the standard model. Remarkably, this scenario also predicts small deviations from flavor-blindness originating from virtual effects of Higgs/top compositeness at $\sim O(10) \mathrm{TeV}$, with subdominant resonance decays into Higgs/top-rich final states, giving the LHC an early "preview" of the nature of the resolution of the hierarchy problem. Discoveries of this type at LHC Run 2 would thereby anticipate (and set a target for) even more explicit explorations of Higgs compositeness at a $100 \mathrm{TeV}$ collider, or for next-generation flavor tests.

KEywords: Phenomenology of Field Theories in Higher Dimensions

ARXIV EPRINT: 1608.00526 


\section{Contents}

1 Introduction $\quad 1$

2 Model with one intermediate brane $\quad 9$

2.1 Parameters 9

$\begin{array}{ll}2.2 \text { Spectrum } & 10\end{array}$

2.3 Couplings 11

$\begin{array}{lll}2.3 .1 & \text { Radion/dilaton } & 11\end{array}$

$\begin{array}{lll}2.3 .2 & \text { Spin-1/Gauge KK } & 13\end{array}$

$\begin{array}{lll}\text { 2.3.3 Spin-2/Graviton KK } & 18\end{array}$

2.4 Extended Bulk Gauge Symmetries/Dual to PNGBs of Vector-Like Confinement 20

$\begin{array}{lll}3 & \text { Phenomenology } & 21\end{array}$

3.1 Radion/dilaton $\quad 22$

3.2 Spin-1 composite 23

3.2.1 Current bounds in flavor-universal limit 23

3.2.2 Probing top/Higgs compositeness 25

3.3 Spin-2 composite 28

4 Model with two intermediate branes 28

5 Conclusions/outlook $\quad 30$

A Details of choice of parameters $\quad 31$

A.1 Matching at the intermediate/Higgs brane 31

$\begin{array}{lll}\text { A.2 Implications of above matching } & 32\end{array}$

B Two dilaton system $\quad 33$

\section{Introduction}

The scenario of Higgs compositeness [1] offers a powerful resolution to the Hierarchy Problem. The Standard Model (SM) Higgs degrees of freedom remain much lighter than the Planck scale in the face of radiative corrections because they are only assembled at $\sim \mathrm{TeV}$ scale, as tightly bound composites of some new strongly interacting "preons". This is in close analogy to how the ordinary charged pion remains much lighter than the Planck scale in the face of QED radiative corrections, by being assembled as a quark-gluon composite at $\sim \mathrm{GeV}$. But despite the simple plot, composite Higgs dynamics is notoriously difficult 
to model in detail because it requires understanding a new strongly-coupled dynamics, operating outside perturbative control.

Remarkably, Higgs compositeness has an alternate "dual" formulation (for the duality of gravity and bulk gauge fields, see, for example, [2]; [3]; for duality of bulk fermions, [4]; $[5,6])$ in the form of "warped" higher-dimensional theories of Randall-Sundrum type $[7,8]$, related to the purely $4 \mathrm{D}$ formulation via the famous AdS/CFT correspondence [9]. In the warped framework there can exist a regime of weakly-coupled higher-dimensional effective field theory, allowing more detailed phenomenological modeling as well as a prototype for UV completion, say within string theory $[10,11]$. Figure 1 shows a schematic representation of particle physics in the simplest such setting, with a single microscopic extra-dimensional interval. The SM is now fundamentally 5-dimensional (for reviews of warped bulk SM, see, for example [12-16]), but its lightest modes appear as the familiar 4D SM particles, with phenomenological properties deriving from their extra-dimensional wavefunctions. In particular, the SM fermions naturally have disparate wavefunctions, which lead to an attractive mechanism for the origin of SM flavor structure, AdS/CFT dual to the robust mechanism of Partial Compositeness ([17]; for AdS dual of this idea, see [4]).

On top of the lightest modes are Kaluza-Klein (KK) excitations of the SM (figure 2), which effectively cut off quantum corrections to the Higgs mass and electroweak symmetry breaking (EWSB). Naturalness then implies that these KK states should have masses of the order TeV scale. ${ }^{1}$ This is the basis of ongoing LHC searches for KK-excited tops and bottoms ("top partners") and KK gauge bosons and spin-2 KK gravitons. Because of their strong extra-dimensional wavefunction-overlap with the top quark and Higgs, these KK resonances predominantly decay to $t, h, W_{L}, Z_{L}$ [18]. From the viewpoint of $4 \mathrm{D}$ Higgs compositeness, the KK excitations are simply other composites of the same preons inside the Higgs (and the closely-related top quark).

Lower-energy experiments are also sensitive to KK states via their virtual exchanges. Electroweak precision tests, now including the rapidly developing body of precision Higgs measurements, robustly constrain the KK spectrum, but are still consistent with KK discoverability at the LHC ([19-22]; for a more recent discussion, see, for example, [23]). However, as in the supersymmetric paradigm, the constraints from tests of flavor and $\mathrm{CP}$ violation are extremely stringent. Although the warped extra-dimensional framework (and partial compositeness) enjoys a powerful generalization of the SM GIM mechanism suppressing FCNCs [24-27], it is imperfect. Typically in parameter space flavor and CP constraints imply $M_{\mathrm{KK}} \gtrsim O(10) \mathrm{TeV}$ for the KK threshold [28-31]!

What are we to make of this situation? While flavor and CP tests have very high virtual reach for the warped/composite scenario, they do not appear as robust as electroweak constraints. It is indeed plausible that a more refined mechanism for flavor structure is occurring within Higgs compositeness so as to relax the bounds significantly, and admit KK states within LHC reach (for models using flavor symmetries for relaxing the bounds,

\footnotetext{
${ }^{1}$ An elegant realization in warped extra dimension of the composite Higgs mechanism, i.e., where it is a PNGB like the pion, is via gauge-Higgs unification [5, 6]. It is in this case that the cutoff of Higgs quantum corrections is the KK scale. However, this aspect plays little role in this paper. So, for brevity, we simply suppress this extra structure of the Higgs field.
} 


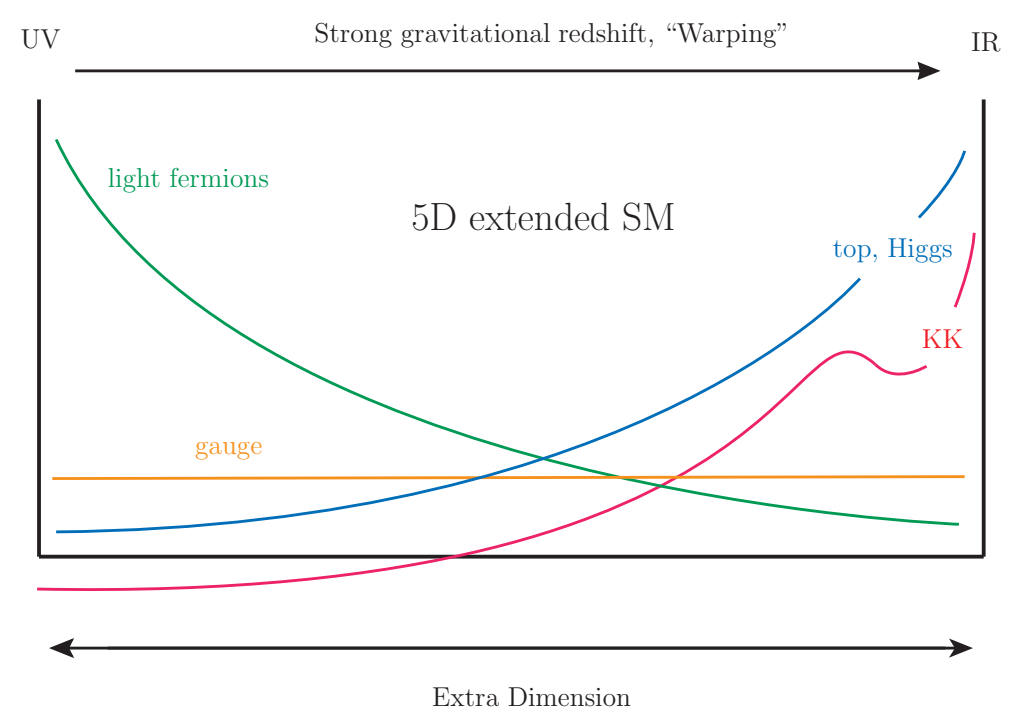

Figure 1. Minimal RS1 model with SM fields in bulk. Also shown are schematic shapes of extradimensional wavefunctions for various particles (zero modes/SM and a generic KK mode).

see, for example, [32]; [33] in 5d; [34-36]; [37] in 4d). Because of this, it is imperative that LHC experiments continue to search for KK resonances along the lines of figure 1 and 2, in tandem with ongoing low-energy searches for new sources of flavor and CP violation. But it is also possible that the hierarchy problem is imperfectly solved by Higgs compositeness at a scale $\gtrsim O(10) \mathrm{TeV}$, leaving a Little Hierarchy Problem between $\sim O(10)$ and $\sim O(1) \mathrm{TeV}$. We simply do not understand fundamental physics and the principle of Naturalness underlying the SM hierarchy problem deeply enough to know if they should reliably predict the threshold of new physics to better than a decade in energy. Of course, such a possibility leads to the practical problem that $M_{\mathrm{KK}} \gtrsim O(10) \mathrm{TeV}$ is outside LHC reach and yet frustratingly close! (It is noteworthy however that such new physics is might be within reach of proposed $100 \mathrm{TeV}$ colliders).

In this paper, we will pursue the scenario of Higgs compositeness at $\gtrsim O(10) \mathrm{TeV}$. This straightforwardly suppresses all virtual KK-mediated electroweak, flavor and $\mathrm{CP}$ violating effects enough to be robustly consistent with all precision experiments to date. But we will ask what natural forms of new physics might lie within $L H C$ reach if we go beyond the minimal structure of figure 1 and 2 , without reintroducing conflict with precision tests. We can think of such non-minimal physics lying below the scale at which the hierarchy problem is solved as "vestiges of naturalness". If the LHC cannot reach the states central to solving the dominant part of the hierarchy problem (such as KK tops), the search for light vestiges, related to the central players but not among them, are the best hope for the LHC.

In particular, we study literally a straightforward extension of figure 1 which exploits the fact that different types of fields can propagate different amounts into the IR of a warped extra dimension, as schematically depicted in figure 3. For simplicity, we focus on three categories of fields: (i) SM matter, including the Higgs, (ii) gauge fields, (iii) gravity. Gravity is the dynamics of all spacetime and therefore must be present in the 


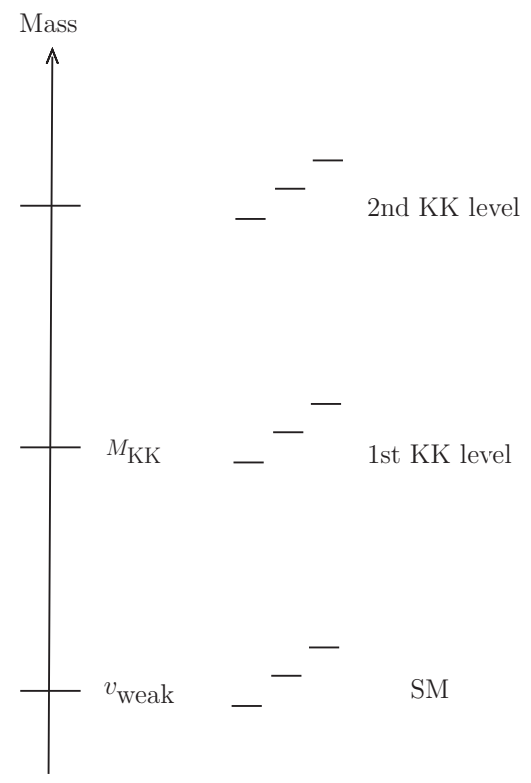

Figure 2. General spectrum of model of figure 1.

entire length of the extra dimension in the form of 5D General Relativity. Gauge fields and matter can however reside in a smaller region. Matter fields can live in an even smaller region of the extra dimension than the gauge fields, but not the other way around because charged matter always radiate gauge fields. This explains the ordering shown in figure 3 . The different regions are separated by "3-branes", $(3+1)$-dimensional defects in the 5D spacetime. Figure 3 is a simple, robust and interesting generalization of the minimal structure of figure 1, 2. A quite different proposal using an intermediate brane in warped spacetime was made in [38] in the context of explaining $750 \mathrm{GeV}$ diphoton excess at the LHC [39, 40]. Different matter fields propagating from the UV brane to different intermediate branes were studied in [41, 42]. Also, a set-up with (only) two branes, but a departure from pure AdS near the infrared brane, can result in the Higgs profile being peaked a bit away from the IR brane [43].

The new physics to the IR of Higgs compositeness is (AdS/CFT dual to) that of "Vectorlike Confinement", proposed in references [44-46] as a phenomenologically rich structure that is remarkably safe from precision tests, and is a natural candidate for a light vestige of a more general dynamics that solves the hierarchy problem. In the framework of figure 3, vectorlike confinement incarnates as the extension of the IR of the extra dimension beyond figure 1, resulting in different KK thresholds for matter, gauge fields and gravity as depicted schematically in figure 4. A simple but important result we will demonstrate is that the Goldberger-Wise (GW) mechanism [47] for brane/radion stabilization very naturally results in "little" hierarchies $M_{\mathrm{KK} \text { matter,Higgs }} \geq M_{\mathrm{KK}_{\text {gauge }}} \geq M_{\mathrm{KK}_{\mathrm{grav}}}$.

From the purely 4D perspective of strong dynamics, the sequence of KK thresholds, $M_{\mathrm{KK}_{\text {matter,Higgs }}} \geq M_{\mathrm{KK} \text { gauge }} \geq M_{\mathrm{KK} \text { grav }}$, is dual to a sequence of strong confinement scales (for non-supersymmetric versions, see, for example, [48]; for supersymmetric cases, see, for example, [49]), $\Lambda_{\text {Higgs }} \geq \Lambda_{\text {meson }} \geq \Lambda_{\text {glueball }}$. Over the large hierarchy from the far 


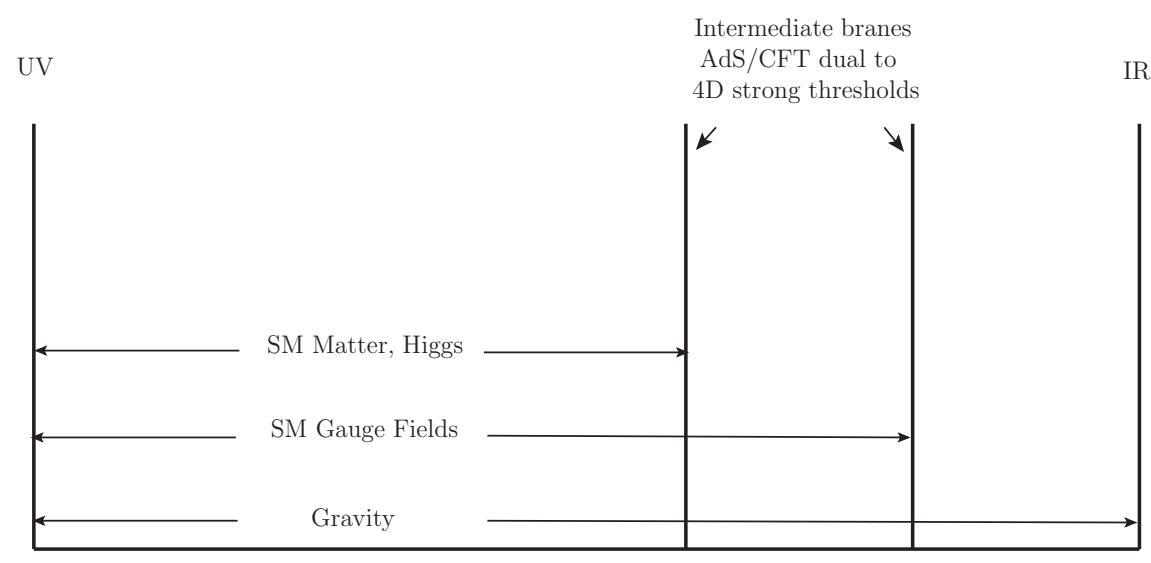

Figure 3. Model with two intermediate branes/thresholds.

UV (the Planck or unification scale) down to $\Lambda_{\text {Higgs }}$ the strong dynamics is only slowly evolving. At $\Lambda_{\text {Higgs }}$ the strong dynamics confines "preons" into composites, among which is the light SM-like Higgs. This is analogous to the emergence of pions and heavier hadrons as composites of quarks and gluons upon QCD confinement. But unlike QCD, the strong dynamics does not end at this point, but rather is reorganized into a new set of strongly interacting preons, now approximately decoupled from Higgs and flavor physics. The IR preons do however carry SM gauge charges.

At $\Lambda_{\text {meson }}$ there is a second stage of preon confinement, into "mesons" also carrying SM gauge charges. Without direct couplings to the Higgs and SM fermions, this second stage of confinement does not break the SM electroweak chiral symmetries, hence the name "vectorlike" confinement. Again, the strong dynamics need not end at this threshold, but can continue with a set of far-IR SM-neutral preons, which ultimately confine into SMneutral "glueballs" at $\Lambda_{\text {glueball }}$.

Since the new physics below $\Lambda_{\text {Higgs }}$ couples to the SM states predominantly via flavorblind gauge forces, it is naturally safe from the host of electroweak, flavor and CP tests. Phenomenologically, production and decay of the new states below $\Lambda_{\text {Higgs }}$ will be mediated by on- and off-shell SM gauge bosons. It is very important that experiments search broadly for this kind of physics. In this way, vectorlike confinement appears as set of "aftershocks" of Higgs compositeness, immune to earlier detection but plausibly lying within grasp of the LHC. We will study several aspects of this strongly motivated scenario in this paper.

In references [44-46], vectorlike confinement was modeled on QCD-like dynamics as the simplest way of illustrating the rich possibilities, using real-world understanding of the strong interactions to stay in non-perturbative theoretical control. A feature of these models is that they typically contain several pseudo Nambu-Goldstone bosons (PNGBs) in the IR of the new physics related to the large chiral symmetry, which can dominate the phenomenology. ${ }^{2}$ However, the specific phenomenological implications are model-dependent. Although QCD-like dynamics do not have a very useful AdS/CFT dual extra-dimensional

\footnotetext{
${ }^{2}$ For recent applications of vector-like confinement for explaining the $750 \mathrm{GeV}$ diphoton excess at the LHC, see, for example, the early references [50-55].
} 


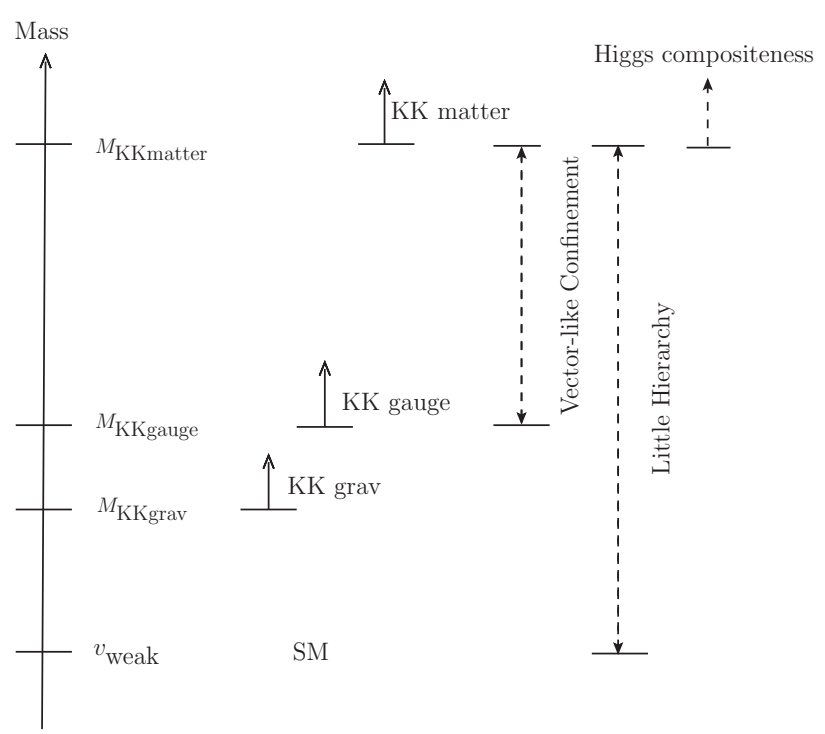

Figure 4. Full spectrum of model of figure 3 .

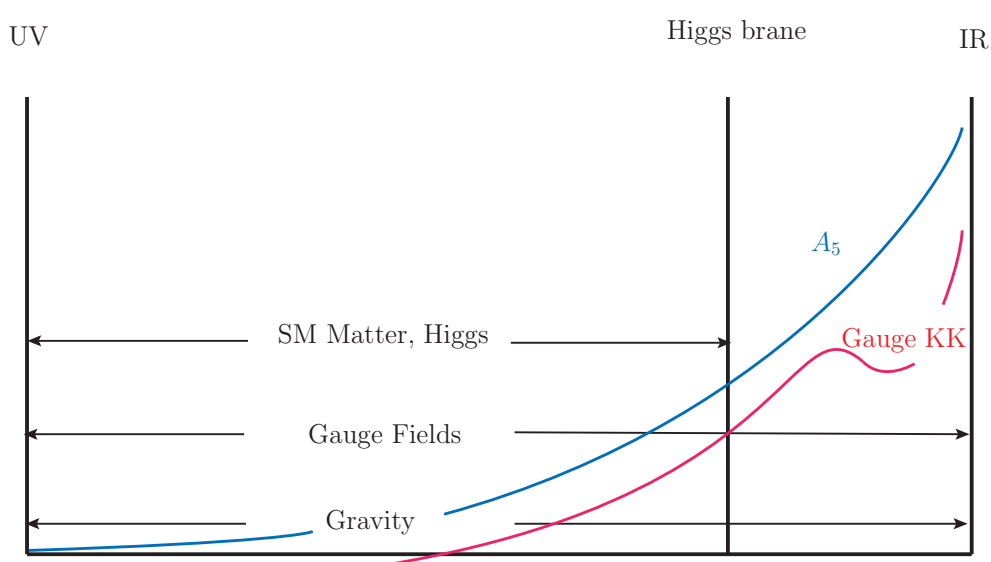

Figure 5. Model with an extended gauge group beyond SM and one intermediate brane, resulting in some number of $A_{5} 4 \mathrm{D}$ scalars dual to composite PNGB's.

description, they are in the same "universality class" as extra-dimensional models of the type depicted in figure 5, where the 5D gauge group is extended beyond the SM. If UV and IR boundary conditions break some of the gauge symmetry generators, they result in physical extra-dimensional components of the gauge field, " $A_{5}$ ", which are $4 \mathrm{D}$ scalars, AdS/CFT dual to PNGB's [5]. ${ }^{3}$ We will return to study this class of vectorlike confining physics more closely in future work. Unlike in QCD-like constructions, in warped $5 \mathrm{D}$ effective field theory we can suppress the existence of $A_{5}$ 's by construction, allowing us to focus on other possibilities for the new phenomenology.

\footnotetext{
${ }^{3}$ Such states tend to be lighter than the typical KK scale and thus can be within LHC reach even in the minimal model of figure 1 with the IR brane at the flavor/CP bound of $\sim O(10) \mathrm{TeV}[56,57]$.
} 
One focus of this paper will be the possibility that lightest new states are the universal ones arising from 5D General Relativity, the scalar "radion" measuring the (dynamical) size of the final IR segment of the extra-dimensional interval, and spin-2 KK gravitons. These are the hallmarks of warped extra-dimensional physics. Via the AdS/CFT correspondence these states are dual to special "glueballs" interpolated by the conserved energy-momentum tensor of the strong dynamics, the universal composite operator of any quantum field theory. In particular, this symmetric tensor naturally interpolates spin-2 glueballs dual to KK gravitons, while its Lorentz-trace interpolates the "dilaton", a glueball dual to the radion. We will derive and discuss their phenomenological implications, pointing out (i) when they are likely to be the first discovered new states beyond the SM, (ii) their special distinguishing features and the contrast with more QCD-like vectorlike confinement and other beyond-SM physics, (iii) how we can experimentally test whether the new physics is well-described by higher-dimensional dynamics.

In table 1, we highlight a couple of signals from the gravity sector, namely radion in the model with one intermediate brane of figure 5 and KK graviton in the model with two intermediate branes of figure 3: further details will be provided in the relevant parts of the paper. For now, it is noteworthy that the decays in these cases dominantly occur to pairs of SM gauge bosons, cf. top/Higgs playing this role in the minimal model of figure 1. Also, we see that radion and KK graviton are allowed to be lighter than gauge KK modes. ${ }^{4}$

A second focus of the paper will be connecting the new physics the LHC can discover to the solution of the hierarchy problem beyond its reach. We will show that low-lying KK modes, though mostly decoupled from the Higgs and flavor, will have subdominant decay channels into $t, h, W_{L}, Z_{L}$, the traditional signatures of Higgs compositeness. In this way, the LHC would have a valuable resonance-enhanced "preview" of the solution to the hierarchy problem by compositeness, only fully accessible to more energetic future colliders. In particular, we find that spin-1 KK gauge bosons are well-suited for this task. Note that these are dual to composite vector " $\rho$ " mesons, which arise as a robust feature in the framework of vector-like confinement also.

A representative sample of the above novel probe of top/Higgs compositeness is shown in table 2: we will of course explain in later sections how we obtained these numbers (including assumptions made therein), but let us convey our main message using them for now. We focus on $\mathrm{KK}$ - excited (dual to composite) $Z$ and gluon, where we fix their mass and coupling to light quarks, hence production cross-section (as shown). However, decay branching ratios (BR's) to various final states still vary for the same framework as we vary $\Lambda_{\text {Higgs: }}$ : the left-most column corresponds to the standard composite Higgs model (i.e., single IR brane/scale, figure 1), whereas right extreme is the flavor-blind limit, i.e., Higgs compositeness scale is decoupled, large $\left.\Lambda_{\text {Higgs }}\right)$. Remarkably, we see that decay BR's might be sensitive to $\sim 10-15 \mathrm{TeV}$ Higgs compositeness scale [in the sense that such values of Higgs compositeness scale can result in $\sim O(1)$ deviations from both flavor-blind and standard limits], which is the ball park of the generic lower limit on the Higgs compositeness scale from flavor/CP violation!

\footnotetext{
${ }^{4}$ It might be also possible to make KK graviton lighter than gauge KK using large brane-localized kinetic terms (BKT) for gravity [58]. For recent applications of this idea for explaining the $750 \mathrm{GeV}$ diphoton excess at the LHC using KK graviton, see [59-64]. However, with too large BKT for gravity, the radion might become a ghost [58].
} 


\begin{tabular}{|c|c|c|c|}
\hline \multicolumn{4}{|c|}{ Radion / KK Graviton } \\
\hline & & Radion $(\varphi)$ & KK Graviton \\
\hline & Framework & one intermediate brane (figure 5) & two intermediate branes (figure 3) \\
\hline \multirow{3}{*}{\multicolumn{2}{|c|}{ Parameters }} & inter-KK gravity coupling $=1$ & inter-KK gravity coupling $=3$ \\
\hline & & inter-KK gauge coupling $=3$ & inter-KK gauge coupling $=3$ \\
\hline & & $M_{\mathrm{KKgauge}}=3 \mathrm{TeV} ; m_{\varphi}=1 \mathrm{TeV}$ & $M_{\text {KKgauge }}=3 \mathrm{TeV} ; M_{\text {KKgrav }}=1 \mathrm{TeV}$ \\
\hline \multicolumn{2}{|c|}{$\sigma_{\mathrm{LHC13}}(p p \rightarrow$ Radion / KK Graviton $)$} & $\sim 80 \mathrm{fb}$ & $\sim 3.9 \mathrm{fb}$ \\
\hline \multirow{4}{*}{$\mathrm{BR}$} & $\mathrm{gg}$ & $\sim 95 \%$ & $\sim 95 \%$ \\
\hline & $\mathrm{ZZ}$ & $\sim 1 \%$ & $\sim 1 \%$ \\
\hline & WW & $\sim 3 \%$ & $\sim 3 \%$ \\
\hline & $\gamma \gamma$ & $\sim 0.7 \%$ & $\sim 0.7 \%$ \\
\hline
\end{tabular}

Table 1. Estimates for production cross section (at $\sqrt{s}=13 \mathrm{TeV}$ LHC) and decay BR's of radion (left) and KK graviton (right) for a given choice of framework and parameters. For radion, model with one intermediate brane is considered with radion mass $1 \mathrm{TeV}, M_{\mathrm{KKgauge}}=3 \mathrm{TeV}$, and inter-KK gravity (gauge) coupling of $1(3)$ [composite gravity $\left(g_{\star}^{\text {grav }}\right)$ and gluon $\left(g_{\star}^{\mathrm{QCD}}\right)$ couplings, respectively, which we define in section 2.1]. For KK graviton, we instead considered model with two intermediate branes, in which KK graviton is naturally lighter than KK gauge boson. In this case, both inter-KK couplings are taken to be 3 .

\begin{tabular}{|c|c|c|c|c|}
\hline \multicolumn{5}{|c|}{ KK Z } \\
\hline \multicolumn{5}{|c|}{$\sigma_{\mathrm{LHC} 13}(p p \rightarrow \mathrm{KK} Z) \sim 2.5 \mathrm{fb}$ for $3 \mathrm{TeV}$ mass and inter-KK coupling of 3} \\
\hline $\begin{array}{ll}\text { Final state } & \Lambda_{\text {Higgs }} \\
\end{array}$ & $3 \mathrm{TeV}$ (figure 1 ) & $10 \mathrm{TeV}$ & $15 \mathrm{TeV}$ & $\infty$ \\
\hline di-leptons $(e+\mu)$ & $\sim 0$ & $\gtrsim 6-4 \%$ & $\gtrsim 6 \%$ & $6 \%$ \\
\hline di-bosons (Higgs $/ W / Z$ ) & $65 \%$ & $\sim 0-28 \%$ & $\sim 0-4 \%$ & $4 \%$ \\
\hline di-tops & $35 \%$ & $9-20 \%$ & $9-10 \%$ & $10 \%$ \\
\hline di-jets & $\sim 0$ & $63-36 \%$ & $63-59 \%$ & $59 \%$ \\
\hline \multicolumn{5}{|c|}{ KK Gluon } \\
\hline \multicolumn{5}{|c|}{$\sigma_{\mathrm{LHC} 13}(p p \rightarrow \mathrm{KK}$ gluon $) \sim 151 \mathrm{fb}$ for $3 \mathrm{TeV}$ mass and inter-KK coupling of 3} \\
\hline $\begin{array}{ll}\text { Final state } & \Lambda_{\text {Higgs }} \\
\end{array}$ & $3 \mathrm{TeV}$ (figure 1 ) & $10 \mathrm{TeV}$ & $15 \mathrm{TeV}$ & $\infty$ \\
\hline di-jets (light quarks $+b$ ) & $\sim 0$ & $83-91 \%$ & $86-91 \%$ & $83 \%$ \\
\hline di-tops & $100 \%$ & $17-9 \%$ & $14-9 \%$ & $17 \%$ \\
\hline
\end{tabular}

Table 2. Estimates for decay BR's of KK $Z$ (top) and KK gluon (bottom) for various values of top/Higgs compositeness scale $\left(\Lambda_{\text {Higgs }}\right)$, for fixed spin- 1 mass scale of $3 \mathrm{TeV}$ and inter-KK $Z /$ gluon coupling $\left[g_{\star}^{Z / \text { gluon }}\right.$, which we define in section 2.1] of 3, corresponding to cross-section (at $\sqrt{s}=$ $13 \mathrm{TeV} \mathrm{LHC}$ ) of $\sim 2.5 \mathrm{fb}$ (for KK $Z$ ) and $\sim 151 \mathrm{fb}$ (for KK gluon).

The paper is organized as follows. We begin in section 2 with laying out the structure of the model with gauge and gravity propagating in the same bulk, but matter/Higgs in a subspace, i.e., with the usual UV and IR branes along with a single intermediate brane demarking the matter/Higgs endpoint. In section 3, we then describe salient features of the LHC signals of this framework. In section 4, we discuss more general framework with two 


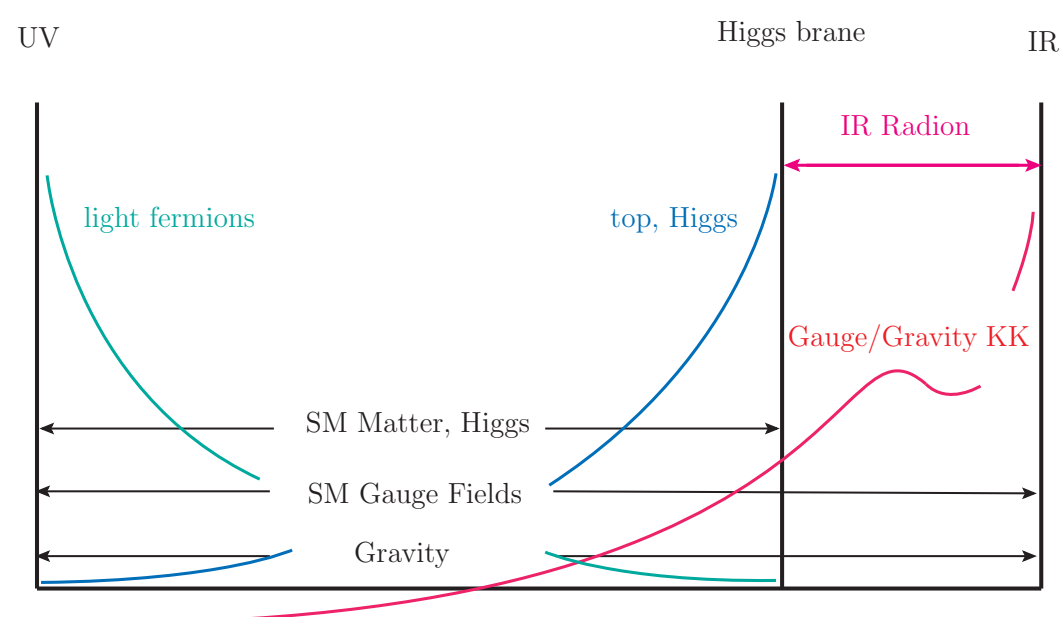

Figure 6. Model with one intermediate brane showing light IR radion degree of freedom.

intermediate branes, in which gravity extends even beyond the gauge bulk. Section 5 provides our conclusions and outlook. Some technical details are relegated to the appendices.

\section{Model with one intermediate brane}

We consider gauge and gravity living in the same bulk starting at the UV brane, with scale $\Lambda_{\mathrm{UV}} \lesssim M_{\mathrm{Pl}}$ and ending at the IR brane, with scale $\Lambda_{\mathrm{IR}}$, which can be as low as $\sim$ a couple of TeV: see figure 6 . In the notation used in section 1 , both $\Lambda_{\text {meson }}$ and $\Lambda_{\text {glueball }}$ are $\sim \Lambda_{\mathrm{IR}}$, which are also (roughly) the gauge and graviton KK scales in the 5D model. For now, we will assume the gauge symmetries to be only the SM throughout the bulk so that we do not have $A_{5}$ 's; we will briefly discuss the latter possibility in section 2.4. The rest of the SM propagates from the UV brane to an intermediate brane (dubbed "Higgs" brane), taken to be $\sim O(10) \mathrm{TeV}$ consistently with (anarchic) flavor bounds. We will discuss more details below, showing that even with contribution from composite states of strong dynamics below $\sim \Lambda_{\text {Higgs }}$, our framework is indeed safe from EW and flavor/CP violation precision tests. As usual, the lighter SM fermions are assumed to be peaked near the UV brane.

\subsection{Parameters}

We use the usual notation where $M_{5}$ is the $5 \mathrm{D}$ Planck scale and $k$ is the AdS curvature scale. The cubic self-coupling of graviton $K K$ modes (or that of one graviton KK to any two modes localized near IR brane, for example, KK gauge) is then given (roughly) by

$$
g_{\star}^{\text {grav }} \equiv \sqrt{\frac{k^{3}}{M_{5}^{3}}}
$$

Also, $g_{\star}^{\text {grav }}$ is dual to coupling of three composites, one of which being spin-2 (and for which we will use the same notation).

Similarly, $g_{5}$ is the (dimension $f u l$ ) $5 \mathrm{D}$ gauge coupling, with the coupling between (three) $4 \mathrm{D}$ modes (one of which is gauge KK) localized near IR brane (or three composites, with 
one being spin-1) given (roughly) by

$$
g_{\star}^{\text {gauge }} \equiv g_{5} \sqrt{k}
$$

As usual, the sizes of both $g_{\star}$ 's are constrained by perturbativity and fitting observed/4D SM couplings (i.e., of zero modes).

However, in the model at hand, there is a new ingredient, namely, the intermediate (Higgs/matter) brane which has tension, i.e., is gravitating, resulting in

(i) $k$ being different on the two sides of this brane and

(ii) a new perturbativity constraint associated with branon (brane-bending) degree of freedom.

We will discuss these issues in detail in appendix A; here we simply summarize. The following choices of couplings (in the far IR) suffice for having a finite regime of validity of $5 \mathrm{D}$ effective field theory (including the branon degree of freedom):

$$
\begin{aligned}
& g_{\star \mathrm{UV}}^{\text {grav }}<g_{\star \mathrm{IR}}^{\text {grav }} \lesssim 3 \\
& g_{\star \mathrm{UV}}^{\text {gauge }} \sim g_{\star \mathrm{IR}}^{\text {gauge }} \sim 3
\end{aligned}
$$

while giving observable signals.

\subsection{Spectrum}

We expect to have two radions (dual to dilatons in the CFT description), roughly corresponding to fluctuations of Higgs brane relative to UV (heavier mode) and that of IR brane relative to Higgs brane. We now work out some of the details of this picture. We first give a schematic review of the GW mechanism in the CFT language for the minimal model of figure 1 [3]. We start in the UV with

$$
\mathcal{L}\left(\Lambda_{\mathrm{UV}}\right) \ni \mathcal{L}_{\mathrm{CFT}}+\lambda \Lambda_{\mathrm{UV}}^{\epsilon} \mathcal{O}_{\mathrm{GW}}
$$

where $\mathcal{O}_{\mathrm{GW}}$ is scalar operator with scaling dimension $(4-\epsilon)$ (with $\epsilon>0$ ): we also use the convention where its naive/engineering dimension is the same so that the coupling constant $\lambda$ above is dimensionless. We assume that $\mathcal{O}_{\mathrm{GW}}$ acquires a VEV in the IR, breaking the conformal symmetry spontaneously; this scale can be thought of as the VEV of the dilaton field (denoted by $\Phi$ of mass dimension +1 ). So, we get the dilaton potential

$$
\mathcal{L}\left(\Lambda_{\mathrm{IR}}\right) \ni\left(\partial_{\mu} \Phi\right)^{2}+\lambda^{\prime} \Phi^{4}+d \lambda \Phi^{4}\left(\frac{\Phi}{\Lambda_{\mathrm{UV}}}\right)^{-\epsilon}
$$

where the second term on the r.h.s. is consistent with conformal symmetry and in the third term, $d$ is an $O(1)$ factor in the interpolation of $\Phi$ by $\mathcal{O}_{\mathrm{GW}}$. Here, we assume that the scaling dimension of $\mathcal{O}_{\mathrm{GW}}$ remains $(4-\epsilon)$ even in the IR and we have dropped subleading terms, i.e. $O\left(\lambda^{2}\left(\frac{\Phi}{\Lambda_{\mathrm{UV}}}\right)^{-2 \epsilon}\right)$. 
Minimizing above potential in the IR, we see that the radius is stabilized, i.e., IR scale is fixed as

$$
\begin{aligned}
\Lambda_{\mathrm{IR}}(\sim\langle\Phi\rangle) & \sim \Lambda_{\mathrm{UV}}\left(-d \frac{\lambda}{\lambda^{\prime}}\right)^{\frac{1}{\epsilon}} \\
& \ll \Lambda_{\mathrm{UV}}, \text { assuming } \epsilon<1
\end{aligned}
$$

where $\sim$ above (and henceforth) indicates validity up to $O(1)$ factors. In particular (and as is well-known), we see that $\epsilon \sim O(0.1)$ (i.e., a mild tuning) together with $\left(-d \lambda / \lambda^{\prime}\right) \sim$ $O[1 /$ (a few $)]$ suffices to generate the enormous Planck-weak hierarchy.

Once again, in the model at hand, we will have two copies of above module, roughly speaking corresponding to the two hierarchies, i.e., $\Lambda_{\text {Higgs }} / \Lambda_{\mathrm{UV}}$ (roughly the usual one) and $\Lambda_{\mathrm{IR}} / \Lambda_{\text {Higgs }}{ }^{5}$ As shown in more detail in the appendix $\mathrm{B}$, the two stabilizations can be done "sequentially", giving a heavy dilaton (mass dictated by $\Lambda_{\text {Higgs }}$ ) and lighter one (mass $\propto \Lambda_{\mathrm{IR}}$ ): for the purpose here (i.e., LHC signals), we will focus on the latter, for which $\Lambda_{\text {Higgs }}$ can be simply taken to be a "fixed/UV" scale. The physical dilaton (denoted by $\varphi$ ) corresponds to fluctuations around the VEV, i.e.,

$$
\Phi \sim \Lambda_{\mathrm{IR}}+a g_{\star}^{\text {grav }} \varphi
$$

where $a$ is an $O(1)$ factor. Plugging this into the above potential, the lighter dilaton mass is then given by [65-68]

$$
m_{\varphi}^{2} \sim \epsilon \lambda^{\prime} \Lambda_{\mathrm{IR}}^{2}
$$

where $\epsilon$ is then (roughly) set (as above) to logarithm of hierarchy (the one relevant here is between Higgs and IR branes) and $\lambda^{\prime}$ is dual, in $5 \mathrm{D}$, to the amount of detuning of IR brane tension. So, to summarize the various scales, we consider the case:

$$
m_{\varphi} \lesssim \Lambda_{\mathrm{IR}} \ll \Lambda_{\text {Higgs }}
$$

\subsection{Couplings}

\subsubsection{Radion/dilaton}

Once again, we treat the separation between UV and Higgs brane to be fixed, thus reducing the (light) radion/dilaton analysis to the usual minimal case with only two branes. We then simply drop the label "IR" on dilaton and $\mathcal{O}_{\mathrm{GW}}$.

Coupling to SM gauge fields. These can be deduced from the running of the SM gauge couplings as follows. We start with value $g_{\mathrm{UV}}$ at $\Lambda_{\mathrm{UV}}$ and pass through various thresholds all the way to $M_{Z}[69]$ :

$$
\begin{aligned}
\frac{1}{g_{\mathrm{SM}}^{2}} \approx & \frac{1}{g_{\mathrm{UV}}^{2}}+b_{\text {strong UV }} \log \left(\frac{\Lambda_{\mathrm{UV}}}{\Lambda_{\text {Higgs }}}\right)+b_{\text {strong IR }} \log \left(\frac{\Lambda_{\mathrm{Higgs}}}{\Lambda_{\mathrm{IR}}}\right) \\
& +\left(b_{\mathrm{SM}}-b_{\text {top, Higgs }}\right) \log \left(\frac{\Lambda_{\mathrm{UV}}}{\Lambda_{\text {Higgs }}}\right)+b_{\mathrm{SM}} \log \left(\frac{\Lambda_{\text {Higgs }}}{M_{Z}}\right)
\end{aligned}
$$

\footnotetext{
${ }^{5}$ Note that we envisage the new, second hierarchy to be at most $O(10)$, i.e., it is (much) smaller than the usual/first one, thus requiring an even more natural value of $\epsilon$, i.e., $\sim 1 /$ a few , cf. $\sim O(0.1)$ for the Planck-weak case.
} 
where $b_{\text {strong UV (IR) }}$ are the contributions of UV and IR 4D strong dynamics (including, in the former case, the SM top quark and Higgs, which are composites), respectively, to the running of the SM gauge coupling and $b_{\mathrm{SM}}$ is the usual SM contribution.

We expect

$$
\begin{aligned}
b_{\text {strong }} & =\frac{O\left(N_{\text {strong }}\right)}{16 \pi^{2}} \\
& \sim \frac{1}{\left(g_{\star}^{\text {gauge }}\right)^{2}}
\end{aligned}
$$

where in second line, we have used the standard large- $N$ relation that coupling of three composites, i.e., $g_{\star}^{\text {gauge }}$ (in this case, one being spin-1/gauge) is given by $\sim 4 \pi / \sqrt{N_{\text {strong }}}$. In fact, the $5 \mathrm{D}$ result is:

$$
b_{\text {strong }}=\frac{1}{g_{5}^{2} k}
$$

which (as expected) is a good match to the second line of eq. (2.12) above [using eq. (2.2)].

The dilaton can be considered to be fluctuations around $\mathrm{TeV}$ scale, i.e., $\Lambda_{\mathrm{IR}} \rightarrow$ $\Lambda_{\mathrm{IR}}+a g_{\star}^{\text {grav }} \varphi$ [see eq. (2.8)]. We plug this into the gauge field kinetic term in the form $F_{\mu \nu} F^{\mu \nu} /\left(4 g_{\mathrm{SM}}^{2}\right)$, with $g_{\mathrm{SM}}$ as in eq. (2.11). We thus get, after canonically normalizing the gauge field, the dilaton coupling to SM gauge bosons $[68,70,71]$ :

$$
\begin{aligned}
\delta \mathcal{L} & \sim g_{\mathrm{SM}}^{2} b_{\text {strong }} \varphi F_{\mu \nu} F^{\mu \nu} \frac{g_{\star}^{\text {grav }}}{\Lambda_{\mathrm{IR}}}+\ldots \\
& \sim\left(\frac{g_{\mathrm{SM}}}{g_{\star}^{\text {gauge }}}\right)^{2} \varphi F_{\mu \nu} F^{\mu \nu} \frac{g_{\star}^{\text {grav }}}{\Lambda_{\mathrm{IR}} .}
\end{aligned}
$$

Coupling to top quark/Higgs. For simplicity, we assume that the top quark/Higgs are strictly localized on the Higgs brane, which (as already mentioned) we are treating (effectively) as "UV" brane for the purpose of obtaining couplings of the light radion. In the 5D model, we can couple the Higgs and top quarks to the 5D GW field (used for stabilization) evaluated at the Higgs brane, thereby generating a coupling of radion to the top quark/Higgs. We will work out the size of this induced coupling in the compositeness picture, the above coupling in the $5 \mathrm{D}$ model being dual to:

$$
\delta \mathcal{L}\left(\Lambda_{\text {Higgs }}\right) \sim \frac{\kappa \Lambda_{\text {Higgs }}^{\epsilon}}{\Lambda_{\text {Higgs }}^{4}} \mathcal{O}_{\mathrm{GW}} \mathcal{O}_{t / H}
$$

where $\mathcal{O}_{t / H}$ is an operator (of mass dimension 4) containing top quark and Higgs fields (to be discussed more below). Since $\mathcal{O}_{\mathrm{GW}}$ obtains a VEV at scale $\Lambda_{\mathrm{IR}}$ (fluctuations around which correspond to the dilaton), we can interpolate it in the IR as

$$
\mathcal{O}_{\mathrm{GW}} \sim \Lambda_{\mathrm{IR}}^{3-\epsilon} g_{\star \mathrm{IR}}^{\text {grav }} \varphi
$$

i.e., (as above) we can choose derivatives to not appear on $\varphi$, which implies that we must allow the most general form of $\mathcal{O}_{t / H}$ (i.e. we cannot integrate by parts to get rid of derivatives on top quark and Higgs fields):

$$
\mathcal{O}_{t / H} \ni \bar{t} \not \partial t-\left(\partial^{\mu} \bar{t}\right) \gamma_{\mu} t+c_{1} y_{t} \bar{t} t H+c_{2}\left(\partial_{\mu} H^{\dagger}\right) \partial^{\mu} H+c_{3} H^{\dagger} \square H+\frac{\left(y_{t}, g_{\mathrm{EW}}\right)^{2} \Lambda_{\mathrm{Higgs}}^{2}}{16 \pi^{2}} H^{\dagger} H
$$

where $c$ 's are independent/arbitrary coefficients. 
Let us consider dilaton decay from each term in turn. A quick, explicit computation shows that amplitude for $\varphi \rightarrow \bar{t} t$ from the top quark "kinetic" ${ }^{6}$ term in $\mathcal{O}_{t / H}$ is $\propto m_{t}$ : a simple argument based on angular momentum conservation for scalar decay into a fermionantifermion pair shows that it must be so. So, the first two terms actually contribute similarly to the third term, i.e., "mass" term (where we have included $y_{t}$, i.e., SM top Yukawa, as flavor spurion in the power counting).

On the other hand, for $\varphi \rightarrow H^{\dagger} H$, i.e., decay into scalars, there is no such constraint from angular momentum conservation: indeed, we explicitly find that kinetic term for $H$ gives amplitude $\propto p_{H 1}$. $p_{H 2} \approx m_{\varphi}^{2} / 2$ (in the limit of $m_{H} \ll m_{\varphi}$ ). Note that contribution of the $\square H$ term (for on-shell $H$ ) is $\propto m_{H}^{2}$, i.e., actual mass term, which is $\ll m_{\varphi}^{2}$, thus is sub-dominant to the contribution of the Higgs kinetic term. In the last term in $\mathcal{O}_{t / H}$, we have assumed that the SM Higgs complex doublet $H$ is a PNGB so that its "mass squared" is SM loop factors smaller than $\Lambda_{\text {Higgs }}^{2}$. Given our choices of $\Lambda_{\text {Higgs }} \sim O(10) \mathrm{TeV}$ and $\Lambda_{\mathrm{IR}} \sim$ a few $\mathrm{TeV}$, we see that this contribution is - roughly and numerically - comparable to that from the Higgs kinetic term.

So, we can just keep top quark mass and Higgs kinetic terms in $\mathcal{O}_{t / H}$ above. We then get

$$
\delta \mathcal{L}\left(\Lambda_{\mathrm{IR}}\right) \sim \kappa\left(\frac{\Lambda_{\mathrm{IR}}}{\Lambda_{\mathrm{Higgs}}}\right)^{4-\epsilon} g_{\star \mathrm{IR}}^{\text {grav }} \frac{\varphi}{\Lambda_{\mathrm{IR}}}\left[m_{t} \bar{t} t+\left(\partial_{\mu} H\right)^{\dagger} \partial^{\mu} H\right]
$$

which gives a (much) smaller decay width for dilaton into top/Higgs as compared to SM gauge bosons in final state. ${ }^{7}$

We conclude from the above analyses that the production of the radion/dilaton is dominated by gluon fusion; dilaton decays mostly to two SM gauge bosons, all via eq. (2.14).

\subsubsection{Spin-1/Gauge KK}

We focus here on the lightest spin- 1 composite, denoted by $\tilde{\rho}$ (reserving $\rho$ for the mass eigenstate: see below).

Flavor universal coupling. The flavor universal part of coupling of $\rho$ (to matter/Higgs fields) is given by a generalization of the well-known phenomenon of $\gamma-\rho$ mixing from QCD (for simplified discussion — using elementary-composite sectors — see, for example,[72]) (see also figure 7), which we briefly review here.

We start with the kinetic and mass terms

$$
\begin{aligned}
\mathcal{L} \ni & -\frac{1}{4}\left(\tilde{\rho}^{\mu \nu} \tilde{\rho}_{\mu \nu}+F^{\text {elem } \mu \nu} F_{\text {elem } \mu \nu}\right) \\
& +\frac{1}{2} \Lambda_{\mathrm{IR}}^{2}\left[\tilde{\rho}_{\mu} \tilde{\rho}^{\mu}-2 \frac{g_{\text {elem }}}{g_{\star}^{\text {gauge }}} \tilde{\rho}^{\mu} A_{\mu}^{\text {elem }}+\left(\frac{g_{\text {elem }}}{g_{\star}^{\text {gauge }}}\right)^{2} A_{\mu}^{\text {elem }} A^{\text {elem } \mu}\right] \\
& +g_{\text {elem }} \bar{q} A_{\mu}^{\text {elem }} \gamma^{\mu} q+g_{\star}^{\text {gauge }} \bar{\psi} \tilde{\rho}_{\mu} \gamma^{\mu} \psi
\end{aligned}
$$

\footnotetext{
${ }^{6}$ Quotes are used here since these are actually multiplied by $\varphi$.

${ }^{7}$ We have checked that other possible contributions to the radion couplings to top/Higgs are comparable to or smaller than the above.
} 


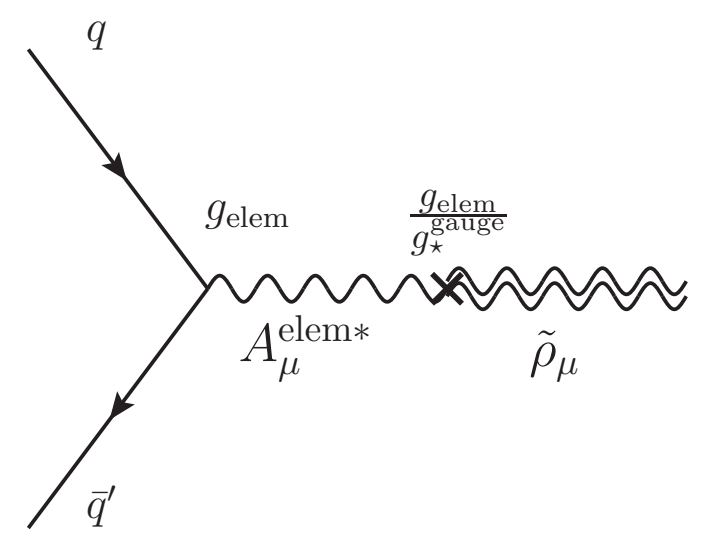

Figure 7. Universal spin-1 couplings via elementary-composite mixing (generalization of wellknown $\gamma-\rho$ mixing).

where $A_{\mu}^{\text {elem }}$ denotes gauge field external to the $4 \mathrm{D}$ strong dynamics (thus often called "elementary"): all SM matter (fermions and Higgs boson, denoted generically by $q$ above) couple to it with strength $g_{\text {elem}}$. Similarly, all composite fermions of strongly coupled sector are denoted by $\psi$ and composite vector meson $\tilde{\rho}_{\mu}$ couples to them with strength $g_{\star}^{\text {gauge }}$. Note that the second term in the second line of eq. (2.19), $\sim \tilde{\rho}_{\mu} A_{\mu}^{\text {elem }}$, is obtained by starting from $A_{\mu}^{\text {elem }} J_{\text {strong IR }}^{\mu}$ and then using the usual interpolation for (the lightest) spin-1 composites ( $\rho$ mesons):

$$
J_{\text {strong IR }}^{\mu} \sim \frac{\Lambda_{\mathrm{IR}}^{2}}{g_{\star}^{\text {gauge }}} \rho_{\mathrm{IR}}^{\mu} .
$$

As we will see, even though the above mass terms break elementary gauge symmetry, there is a residual gauge invariance (corresponding to a massless field) which we identify with the final SM gauge symmetry [72]. We diagonalize the mass terms by defining the physical states (admixtures of $\tilde{\rho}$ and $A_{\mu}^{\text {elem }}$ ):

$$
\begin{aligned}
A_{\mu} & =\cos \theta A_{\mu}^{\mathrm{elem}}+\sin \theta \tilde{\rho}_{\mu} \\
\rho_{\mu} & =\cos \theta \tilde{\rho}_{\mu}-\sin \theta A_{\mu}^{\text {elem }}
\end{aligned}
$$

and

$$
\sin \theta=\frac{g_{\text {elem }}}{\sqrt{g_{\text {elem }}^{2}+g_{\star}^{\text {gauge } 2}}} .
$$

This gives

$$
\begin{aligned}
\mathcal{L} \ni & -\frac{1}{4}\left(\rho^{\mu \nu} \rho_{\mu \nu}+F^{\mu \nu} F_{\mu \nu}\right) \\
& +\frac{1}{2} \Lambda_{\mathrm{IR}}^{2} \rho^{\mu} \rho_{\mu}+g_{\mathrm{SM}} \bar{\psi} A^{\mu} \gamma_{\mu} \psi+g_{\star}^{\text {gauge }} \bar{\psi} \rho^{\mu} \gamma_{\mu} \psi \\
& +g_{\mathrm{SM}} \bar{q} A^{\mu} \gamma_{\mu} q+\frac{g_{\mathrm{SM}} g_{\mathrm{elem}}}{g_{\star}^{\text {gauge }}} \bar{q} \rho^{\mu} \gamma_{\mu} q+\cdots
\end{aligned}
$$


where the last term is the (universal) coupling of SM fermions to $\rho$. Also, as anticipated above, $A_{\mu}$ is massless (thus corresponds to the SM gauge field), with

$$
g_{\mathrm{SM}}=\frac{g_{\star}^{\text {gauge }} g_{\text {elem }}}{\sqrt{g_{\text {elem }}^{2}+g_{\star}^{\text {gauge } 2}}}
$$

being the SM gauge coupling. Henceforth, we will assume $g_{\text {elem }} \ll g_{\star}^{\text {gauge }}$ so that

$$
g_{\mathrm{SM}} \approx g_{\mathrm{elem}}
$$

and coupling of SM fermions to $\rho$ is $\approx g_{\mathrm{SM}}^{2} / g_{\star}^{\text {gauge }}$.

Couplings to radion/dilaton. As discussed above, couplings of dilaton/radion can be obtained by using it as a "compensator" for $\Lambda_{\mathrm{IR}}$, giving eq. (2.14) from dependence of $g_{\mathrm{SM}}{ }^{8}$ on $\Lambda_{\text {IR }}$ (via RG evolution of the gauge coupling) and a coupling to two $\tilde{\rho}$ 's (which gets converted mostly into two $\rho$ 's):

$$
\begin{aligned}
\delta \mathcal{L} & \sim \Lambda_{\mathrm{IR}}^{2} \tilde{\rho}_{\mu} \tilde{\rho}^{\mu} \\
& \rightarrow \Phi_{\mathrm{IR}}^{2} \tilde{\rho}_{\mu} \tilde{\rho}^{\mu} \\
& \ni g_{\star \mathrm{IR}}^{\text {grav }} \Lambda_{\mathrm{IR}} \varphi \rho^{\mu} \rho_{\mu}
\end{aligned}
$$

which however is not relevant for collider signals. Note that using $\gamma-\rho$ mixing in first line of eq. (2.27), one naively obtains couplings of $\varphi$ to $A_{\mu} A^{\mu}$ or $A_{\mu} \rho^{\mu}$; however, after properly adding contributions from the other two terms in the second line of eq. (2.19), we can see that these terms vanish.

In addition, after radius stabilization/explicit breaking of conformal symmetry, we get a mixed coupling of dilaton, i.e., to $\rho$ and SM gauge field as follows. In the IR, we can interpolate the GW operator as

$$
\mathcal{O}_{\mathrm{GW}} \ni \Lambda_{\mathrm{IR}}^{-\epsilon} \tilde{\rho}^{\mu \nu} \tilde{\rho}_{\mu \nu} .
$$

Plugging above in eq. (2.4), RG-running down to $\Lambda_{\mathrm{IR}}$ and then promoting $\Lambda_{\mathrm{IR}} \rightarrow \Lambda_{\mathrm{IR}}+$ $a g_{\star \mathrm{IR}}^{\mathrm{grav}} \varphi$, we get

$$
\begin{aligned}
\delta \mathcal{L}\left(\Lambda_{\mathrm{IR}}\right) & \sim \lambda\left(\frac{\Lambda_{\mathrm{IR}}}{\Lambda_{\mathrm{Higgs}}}\right)^{-\epsilon} \tilde{\rho}^{\mu \nu} \tilde{\rho}_{\mu \nu} \\
& \sim \lambda \epsilon g_{\star \mathrm{IR}}^{\mathrm{grav}}\left(\frac{\Lambda_{\mathrm{IR}}}{\Lambda_{\mathrm{Higgs}}}\right)^{-\epsilon} \frac{\varphi}{\Lambda_{\mathrm{IR}}} \tilde{\rho}^{\mu \nu} \tilde{\rho}_{\mu \nu} .
\end{aligned}
$$

Finally, plugging the mass eigenstates from eq. (2.22) into above gives: ${ }^{9}$

$$
\delta \mathcal{L}\left(\Lambda_{\mathrm{IR}}\right) \sim \lambda \epsilon g_{\star \mathrm{IR}}^{\text {grav }}\left(\frac{\Lambda_{\mathrm{IR}}}{\Lambda_{\mathrm{Higgs}}}\right)^{-\epsilon} \frac{g_{\text {elem }}}{g_{\star \mathrm{IR}}^{\text {gauge }}} \rho^{\mu \nu} F^{\mu \nu} \frac{\varphi}{\Lambda_{\mathrm{IR}}} .
$$

\footnotetext{
${ }^{8}$ More precisely, dependence of $g_{\mathrm{SM}}$ on $\Lambda_{\mathrm{IR}}$ originates from dependence of $g_{\text {elem }}$ on $\Lambda_{\mathrm{IR}}$ via the relation $\frac{1}{g_{\mathrm{SM}}^{2}}=\frac{1}{g_{\text {elem }}^{2}}+\frac{1}{g_{\star}^{\text {gauge } 2}}$.

${ }^{9}$ The same procedure also results in couplings of the form $\varphi \rho^{\mu \nu} \rho_{\mu \nu}$ or $\varphi F^{\mu \nu} F_{\mu \nu}$, i.e., corrections to the couplings of dilaton/radion to pairs of SM gauge fields from radius stabilization [68, 71] and $\rho$ 's; however, these are $s u b$-dominant to the pre-existing ones, hence we will not discuss them further.
} 


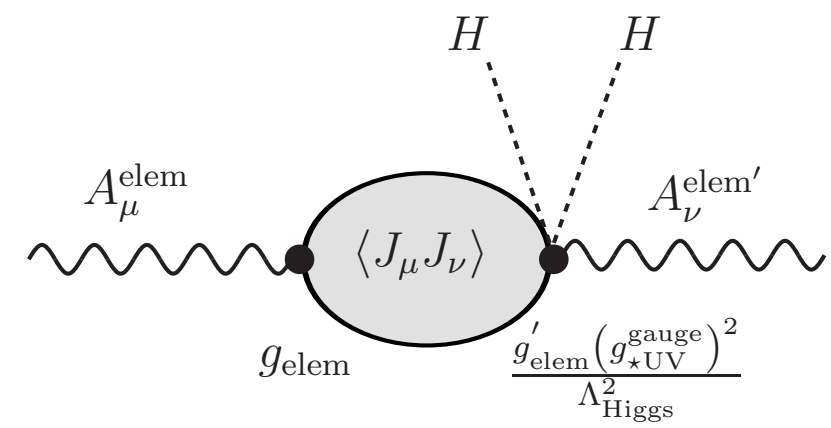

Figure 8. Contribution to the S-parameter from the IR strong dynamics.

From eq. (2.7), here we have $\epsilon \sim 1 / \log \left(\Lambda_{\text {Higgs }} / \Lambda_{\mathrm{IR}}\right) \sim 1 /$ a few, since the relevant hierarchy is $\Lambda_{\mathrm{Higgs}} / \Lambda_{\mathrm{IR}}$ as indicated (again, it is not the large one: $\Lambda_{\mathrm{UV}} / \Lambda_{\mathrm{IR}}$ ), and from this we also see that $\left(\Lambda_{\mathrm{IR}} / \Lambda_{\text {Higgs }}\right)^{-\epsilon}$ is an $O(1)$ factor. Thus, the $\rho$-dilaton-SM gauge boson coupling in eq. (2.30) can be (roughly) comparable to the last term in eq. (2.24), i.e., universal $\rho$ coupling (assuming $g_{\star \mathrm{IR}}^{\text {grav }} \sim 1$ ). Note that decay of $\rho$ to two $\varphi$ (cf. spin-2 below) is not allowed by a combination of Bose-Einstein statistics and angular momentum conservation arguments.

Flavor non-universal couplings to top/Higgs. On the other hand, the flavor nonuniversal part of the $\rho$ couplings (relevant only for top quark/Higgs: negligible for light fermions, at least for LHC signals) arises from

$$
\delta \mathcal{L}\left(\Lambda_{\text {Higgs }}\right) \sim \frac{\left(g_{\star}^{\text {gauge }}\right)^{2}}{\Lambda_{\text {Higgs }}^{2}} J_{\text {strong IR }}^{\mu}\left(\bar{t} \gamma_{\mu} t+H^{\dagger} D_{\mu} H\right)
$$

where this coupling of top/Higgs to IR strong dynamics is generated by integrating out physics of top/Higgs compositeness at scale $\sim \Lambda_{\text {Higgs }}$, with a coupling characteristic of gauge sector of the $U V$ strong dynamics (see appendix B for further explanation of the UV and IR CFT's with stabilization mechanism). This runs down to the IR:

$$
\delta \mathcal{L}\left(\Lambda_{\mathrm{IR}}\right) \sim \frac{\left(g_{\star \mathrm{UV}}^{\text {gauge }}\right)^{2}}{g_{\star \mathrm{IR}}^{\text {gauge }}}\left(\frac{\Lambda_{\mathrm{IR}}}{\Lambda_{\mathrm{Higgs}}}\right)^{2} \rho_{\mathrm{IR}}^{\mu}\left(\bar{t} \gamma_{\mu} t+H^{\dagger} D_{\mu} H\right)
$$

where we have used the interpolation relation of eq. (2.20).

Clearly, the production of $\rho$ at the LHC proceeds via light quark coupling in last term in eq. (2.24), while decays occur via same coupling and that in eq. (2.32) and (2.30), assuming $\varphi$ is lighter than $\rho$.

Electroweak and flavor/CP violation precision tests. The physics of top/Higgs compositeness with characteristic mass scale $\sim \Lambda_{\text {Higgs }}$ (where the UV strong dynamics confines) contributes to EW and flavor/CP violation precision tests. However, as we already indicated at the beginning of section 2, these contributions are safe from experimental constraints for the choice of $\Lambda_{\text {Higgs }} \sim \mathcal{O}(10) \mathrm{TeV}$. Notice that the (small) flavor non-universal 
parts of the couplings of spin- 1 resonances of the $I R$ strong dynamics [see eq. (2.32)] which are suppressed by $\sim \Lambda_{\text {Higgs }}$ - also give contributions (via their virtual exchange) to $\mathrm{EW}$ and flavor/CP violation precision tests. However, as we will show now, such effects are comparable to the direct (albeit still virtual) effects of $\Lambda_{\text {Higgs }}$ scale physics hence are safe/on the edge (just like the latter).

We begin our discussion by considering contributions of IR strong dynamics to precision tests observables using the above non-universal coupling only once, for example, the operator corresponding to the $S$-parameter:

$$
\begin{aligned}
\delta \mathcal{L} & \sim C W_{\mu \nu}^{3} B_{\mu \nu} H^{\dagger} H, \text { with } \\
C & \equiv \frac{g g^{\prime} S}{16 \pi v^{2}},
\end{aligned}
$$

$W_{3}$ and $B$ being the neutral SU(2) and hypercharge gauge fields and $g\left(g^{\prime}\right)$ are the respective gauge couplings. Integrating out physics at and above the scale $\sim \Lambda_{\text {Higgs }}$ generates in the IR effective theory the above operator with coefficient $C_{\mathrm{UV}} \sim g g^{\prime} / \Lambda_{\text {Higgs }}^{2}$ (based on usual, naive dimensional analysis). The contribution from the IR strong dynamics can be obtained by computing the diagram shown in figure 8 . Such a diagram can be generated by sewing together eq. (2.31) (non-universal coupling) and the (universal) coupling $A_{\mu} J_{\text {strong IR }}^{\mu}$ [mentioned below eq. (2.19)], via the common $J_{\text {strong IR }}^{\mu}$

$$
g_{\text {elem }}\left(g_{\star \mathrm{UV}}^{\text {gauge }}\right)^{2} A_{\mu}^{\text {elem }}\left\langle J_{\text {strong IR }}^{\mu} J_{\text {strong IR }}^{\nu}\right\rangle \frac{J_{\nu}^{\mathrm{t} / \mathrm{H}}}{\Lambda_{\text {Higgs }}^{2}} .
$$

The current-current correlator $\left\langle J^{\mu}(p) J^{\nu}(-p)\right\rangle$ contains the piece $\left(\eta^{\mu \nu}-\frac{p^{\mu} p^{\nu}}{p^{2}}\right) p^{2} \log p^{2}$ which contributes to the $S$-parameter operator. We thus find a log-divergence in the $S$-parameter in the theory below $\Lambda_{\text {Higgs }}$. Finally, matching to the $S$-parameter operator and using eq. (2.12) for overall size of correlator, we get $C_{\text {strong IR }} \sim$ $g g^{\prime} \log \left(\Lambda_{\text {Higgs }} / \Lambda_{\text {IR }}\right) / \Lambda_{\text {Higgs }}^{2}{ }^{10}$ As already mentioned above, the total contribution of the IR strong dynamics to $S$-parameter is then comparable to that from physics at $\Lambda_{\text {Higgs }}$. However, there is an important feature we want to emphasize. Namely, the contribution of IR strong dynamics to $S$-parameter shows a mild logarithmic enhancement! This enhancement, however, is not harmful because, with custodial symmetry protection, the constraint from EW precision test on the Higgs compositeness scale in the minimal model of figure 1 can be as low as $\sim 3 \mathrm{TeV}$ [19-23] so that, even with the above enhancement in the extension in figure 6 , the overall size is small enough with $\Lambda_{\text {Higgs }} \sim O(10) \mathrm{TeV}$.

Next, we consider cases where two non-universal couplings are involved, giving (for example) a 4-top quark operator, which after rotation to mass basis for quarks will give flavor-violating effects even for light fermions such as $K-\bar{K}$ mixing [24-31]. Clearly, the contribution of UV strong dynamics to such effects is $\propto 1 / \Lambda_{\text {Higgs }}^{2}$ (just like for $S$-parameter above). For the IR strong dynamics contribution, we combine eq. (2.31) with itself in this

\footnotetext{
${ }^{10}$ We have also checked explicitly that the contribution to the $S$-parameter from the sum over tree-level exchanges of composite resonances (in the $4 \mathrm{D}$ picture with strong dynamics) or gauge KK modes (from the $5 \mathrm{D}$ model) gives a log-divergence.
} 


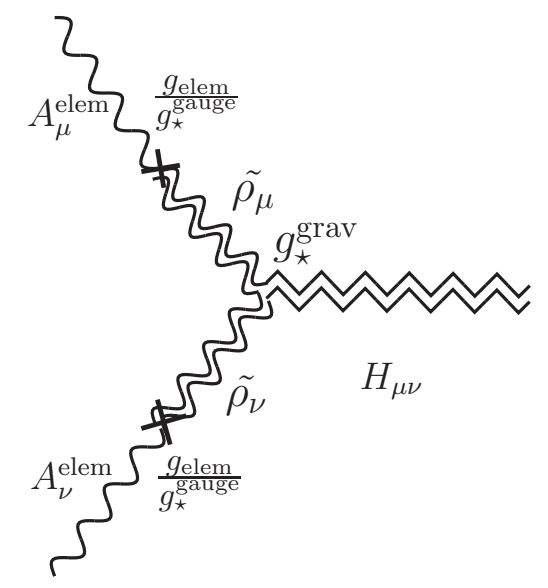

Figure 9. Spin-2 KK graviton couplings to SM gauge bosons.

case. Here, the current-current correlator can instead give a quadratic divergence, which reduces the initial $\sim 1 / \Lambda_{\text {Higgs }}^{4}$ suppression by two powers. That is, the contribution from the entire IR strong dynamics to such flavor/CP violating processes are comparable to that of the physics of the UV strong dynamics, hence safe.

We stress that, for a fixed $\Lambda_{\text {Higgs }}$, the contribution to precision tests from IR strong dynamics is (roughly) independent of $\Lambda_{\mathrm{IR}}$ so that there is no relevant constraint on $\Lambda_{\mathrm{IR}}$ from here; instead the bound on $\Lambda_{\mathrm{IR}}$ is dominated by the direct LHC searches which will be discussed in section 3.2.1.

\subsubsection{Spin-2/Graviton KK}

We denote the composite spin- 2 by $H^{\mu \nu}$. In general, $H^{\mu \nu}$ couples to not only $T_{\mu \nu}$ of composites, but also other possible Lorentz structures built out of the latter fields [73]. Here, for simplicity and because it dominates in warped 5D effective field theory, we will use (only) $T_{\mu \nu}$ as a representative structure (others will anyway give roughly similar size for coupling/amplitude). If experiments show spin structures other than $T_{\mu \nu}$, it would point to strong dynamics without a good 5D dual.

Coupling to SM gauge bosons. The coupling of $H^{\mu \nu}$ to $S M$ gauge bosons is obtained (see figure 9 ) by first coupling it to $\tilde{\rho}$ 's with strength $g_{\star \mathrm{IR}}^{\text {grav }}$ (i.e., a 3-composite vertex), followed by mixing of $\tilde{\rho}$ 's with external gauge field (as outlined above), i.e.,

$$
\begin{aligned}
\delta \mathcal{L}\left(\Lambda_{\mathrm{IR}}\right) & \sim \frac{g_{\star \mathrm{IR}}^{\text {grav }}}{\Lambda_{\mathrm{IR}}} H^{\mu \nu} T_{\mu \nu}^{(\tilde{\rho})} \\
& \rightarrow\left(\frac{g_{\mathrm{SM}}}{g_{\star \mathrm{IR}}^{\text {gauge }}}\right)^{2} \frac{g_{\star \mathrm{IR}}^{\text {grav }}}{\Lambda_{\mathrm{IR}}} H^{\mu \nu} T_{\mu \nu}^{(\text {gauge })} .
\end{aligned}
$$

Coupling to radion/dilaton. In addition, we have the coupling to two dilatons/radions:

$$
\delta \mathcal{L}\left(\Lambda_{\mathrm{IR}}\right) \sim \frac{g_{\star}^{\mathrm{grav}}}{\Lambda_{\mathrm{IR}}} H^{\mu \nu} T_{\mu \nu}^{(\varphi)} .
$$




\begin{tabular}{|c|c|c|}
\hline $\begin{array}{ll}\text { Resonance } & \text { Type of coupling } \\
\end{array}$ & Higgs compositeness-sensitive & flavor-blind \\
\hline radion/dilaton & $\frac{g_{\mathrm{IIR}}^{\text {grav }}}{\Lambda_{\mathrm{IR}}}\left(\frac{\Lambda_{\mathrm{IR}}}{\Lambda_{\mathrm{Higgs}}}\right)^{4-\epsilon}\left[m_{t} \bar{t} t+(\partial H)^{2}\right]$ & $\frac{g_{\star \mathrm{IR}}^{\mathrm{grav}}}{\Lambda_{\mathrm{IR}}}\left(\frac{g_{\mathrm{SMI}}}{g_{\star \mathrm{IR}}^{\text {gauge }}}\right)^{2} F^{\mu \nu} F_{\mu \nu}$ \\
\hline $\mathrm{KK} Z$ & $\frac{\left(g_{\star \mathrm{UVV}}^{\text {gauge }}\right)^{2}}{g_{\star \mathrm{IR}}^{\text {gave }}}\left(\frac{\Lambda_{\mathrm{IR}}}{\Lambda_{\mathrm{Higgs}}}\right)^{2}\left(\bar{t} \gamma^{\mu} t+H^{\dagger} \partial^{\mu} H\right)$ & $\frac{g_{\mathrm{EW}}^{2}}{g_{\star \mathrm{IR}}^{\text {gave }}}\left(\bar{q} \gamma^{\mu} q+\bar{l} \gamma^{\mu} l\right)$ (all generations) \\
\hline KK gluon & $\frac{\left(g_{\mathrm{UV}}^{\text {gauge }}\right)^{2}}{g_{\star \mathrm{IR}}^{\text {gauge }}}\left(\frac{\Lambda_{\mathrm{IR}}}{\Lambda_{\mathrm{Higgs}}}\right)^{2} \bar{t} \gamma^{\mu} t$ & $\frac{g_{\mathrm{QCD}}^{2}}{g_{\star \mathrm{IR}}^{\text {gavge }}} \bar{q} \gamma^{\mu} q($ all generations $)$ \\
\hline KK graviton & $\frac{\left(g_{\star \mathrm{UV}}^{\text {grav }}\right)^{2}}{g_{\star \mathrm{IR}}^{\text {grav }}} \frac{1}{\Lambda_{\mathrm{IR}}}\left(\frac{\Lambda_{\mathrm{IR}}}{\Lambda_{\mathrm{Higgs}}}\right)^{4} T_{\mu \nu}^{(t / H)}$ & $\frac{g_{\star \mathrm{IR}}^{\text {grav }}}{\Lambda_{\mathrm{IR}}}\left(\frac{g_{\mathrm{SM}}}{g_{\star \mathrm{IR}}^{\text {gauge }}}\right)^{2} T_{\mu \nu}^{\text {(gauge })}$ \\
\hline
\end{tabular}

Table 3. Summary of universal and non-universal couplings of various composites for the model with one intermediate brane. $T_{\mu \nu}^{(t / H)}\left(T_{\mu \nu}^{(\text {gauge) }}\right)$ is energy-momentum tensor made of top/Higgs (SM gauge bosons) fields.

Of course, this is relevant for decay of composite spin-2/KK graviton only if $m_{\varphi} \lesssim \Lambda_{\mathrm{IR}} / 2$ and in this case, dominates over other decays: see, for example, [64].

Flavor non-universal coupling (to top/Higgs). Finally, coupling to top quark/Higgs follows from a procedure similar to spin-1 above, i.e., we have

$$
\delta \mathcal{L}\left(\Lambda_{\mathrm{Higgs}}\right) \sim \frac{\left(g_{\star \mathrm{UV}}^{\mathrm{grav}}\right)^{2}}{\Lambda_{\mathrm{Higgs}}^{4}} T^{\mu \nu(t / H)} T_{\mu \nu}^{(\text {strong IR })}
$$

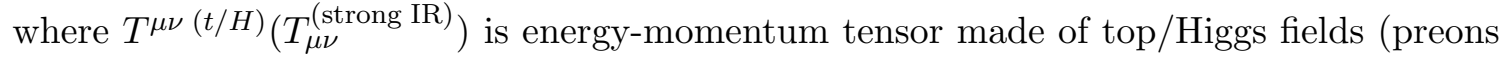
of IR strong dynamics) and this coupling of top/Higgs to IR strong dynamics is generated by integrating out physics at the scale $\sim \Lambda_{\text {Higgs }}$, with a coupling characteristic of gravity sector of the UV strong dynamics. After IR theory hadronizes, eq. (2.37) becomes

$$
\delta \mathcal{L}\left(\Lambda_{\mathrm{IR}}\right) \sim\left(\frac{\Lambda_{\mathrm{IR}}}{\Lambda_{\mathrm{Higgs}}}\right)^{4} \frac{\left(g_{\star \mathrm{UV}}^{\mathrm{grav}}\right)^{2}}{g_{\star}^{\mathrm{grav}}} \frac{H^{\mu \nu}}{\Lambda_{\mathrm{IR}}} T_{\mu \nu}^{(t / H)}
$$

using the interpolation

$$
T_{\mu \nu}^{\text {strong IR }} \sim \frac{\Lambda_{\mathrm{IR}}^{3}}{g_{\star \mathrm{IR}}^{\text {grav }}} H_{\mu \nu}
$$

Production of composite spin-2/KK graviton occurs via coupling to gluons in eq. (2.35). Decays of composite spin-2/KK graviton is dominated by the same couplings, i.e., into all SM gauge bosons and to pair of dilatons via eq. (2.36), assuming $m_{\varphi}<\Lambda_{\mathrm{IR}} / 2$. We give a summary of relevant couplings in table 3 .

Given the above flavor non-universal couplings of KK graviton of the IR strong dynamics (cf. those of gauge KK discussed earlier), it is clear that contributions from KK graviton exchange to precision tests are suppressed compared to those of gauge KK by $\sim E^{2} / \Lambda_{\text {Higgs }}^{2}$, where $E$ is the characteristic (low) energy of the corresponding test. Hence, there is no additional constraint here from the KK graviton sector. 


\subsection{Extended Bulk Gauge Symmetries/Dual to PNGBs of Vector-Like Con- finement}

Relation to vector-like confinement. We can enlarge the bulk gauge symmetries beyond the SM. We then consider breaking them down to the smaller groups (while preserving the SM subgroup of course) on the various branes by simply imposing Dirichlet boundary condition, i.e.,

$$
G_{\mathrm{UV}} \stackrel{\Lambda_{\mathrm{Higgs}}}{\rightarrow} G_{\mathrm{IR}} \stackrel{\Lambda_{\mathrm{IR}}}{\rightarrow} H_{\mathrm{IR}} \supset \mathrm{SM}
$$

where each stage of gauge symmetry breaking delivers (scalar) $A_{5}$ 's, localized at the corresponding brane (including possibly the SM Higgs boson in the first step). Such a framework is shown in figures 5 and 10 . These $A_{5}$ 's are dual to PNGBs arising from spontaneous breakdown of global symmetries of the strong dynamics corresponding to the gauged ones shown in eq. (2.40) [5]. In particular, the 4D physics dual to the last stage of breaking (rightmost bulk in figures 5 and 10), i.e., SM symmetries being unbroken, is known in the literature as vector-like confinement [44-46]. While from the $5 \mathrm{D}$ viewpoint, presence of $A_{5}$ 's seems rather "non-minimal", it is quite natural to have PNGB's in 4D strong dynamics as illustrated by ordinary QCD. In fact, QCD-like strong dynamics was first used to realize the general idea of vector-like confinement.

Note that $A_{5}$ 's are massless at tree-level (in the presence of only the above boundary condition breaking), acquiring a potential via loops, with mass scale being set by corresponding $\Lambda$. Thus they are naturally light, as expected from them being dual to PNGB's. Gauge and graviton KK modes (and even possibly the radion) can then decay into pairs of $A_{5}$ 's, drastically modifying the LHC signals of the gauge and graviton KK (or radion) based only on the couplings shown earlier. In this paper, we take the minimal 5D perspective in assuming that $A_{5}$ 's are absent, cf. the expectation based on QCD-like 4D strong dynamics. Hence, gauge KK will decay dominantly into pairs of SM fermions, while SM gauge bosons will be the search channel for KK graviton and radion, as mentioned earlier. Remarkably, the flexibility afforded by $5 \mathrm{D}$ leads to broader class of models, with more diverse phenomenology than contemplating just 4D QCD-like strong dynamics.

Coupling to two SM gauge bosons. There is an interesting comparison with dilaton/radion that we would like to draw by considering the simplest mechanism for production and decay of (single) $A_{5}$ (dual to PNGB). Namely, PNGB famously has a coupling to two weakly-coupled gauge bosons via the (gauged) Wess-Zumino-Witten term, for example, we have $\pi^{0} F_{\mu \nu} \tilde{F}^{\mu \nu}$ leading to the decay $\pi^{0} \rightarrow \gamma \gamma$ in real-world QCD. This interaction is dual to the one originating for the $A_{5}$ from the Chern-Simons term in the $5 \mathrm{D}$ model (see also discussions in $[50,74])$ :

$$
\begin{aligned}
\mathcal{L}_{5 \mathrm{D}} & \ni K f_{a b c} \epsilon^{M N R S T} A_{M}^{a} F_{N R}^{b} F_{S T}^{c}+\ldots \\
& \sim K f_{a b c} A_{5}^{a} F^{b \mu \nu} \tilde{F}_{\mu \nu}^{c}+\ldots
\end{aligned}
$$

where $a, b, c$ are gauge adjoint indices. 


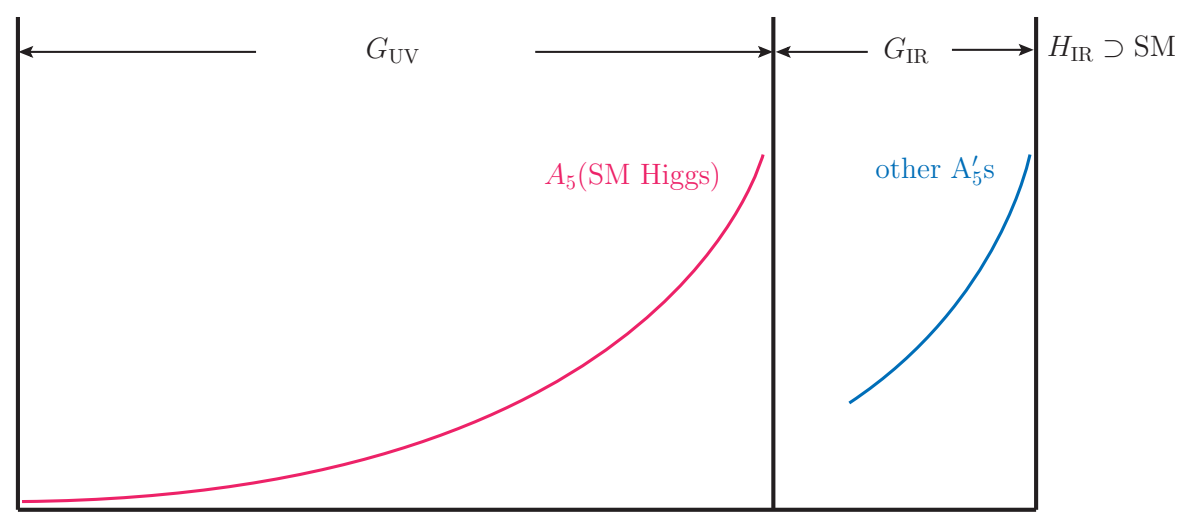

Figure 10. Extended bulk gauge symmetries, with rightmost bulk segment being dual to vector-like confinement.

Crucially, we see that, irrespective of considerations of parity as a fundamental symmetry, the coupling of $A_{5}$ to two SM gauge bosons via Chern-Simons term has "CP-odd" structure, i.e., involves $F_{\mu \nu} \tilde{F}^{\mu \nu}$. This feature is in contrast to the "CP-even" coupling, i.e., to $F_{\mu \nu} F^{\mu \nu}$, of dilaton/radion as we see in eq. (2.14). Let us compare to vector-like confinement, in particular, QCD-like dynamics: this theory respects parity even in the IR and PNGB's are parity-odd (as per the Vafa-Witten theorem $[75,76]$ ), which enforces a coupling to pairs of SM gauge bosons to be to the combination $F_{\mu \nu} \tilde{F}^{\mu \nu}$. However, we see that there is a more general (than parity) argument for such a structure from Chern-Simons term in $5 \mathrm{D}$.

Moreover, the 5D Chern-Simons term is dual to anomalies in global currents of the $4 \mathrm{D}$ strong dynamics, i.e., $K$ of eq. (2.41) - appropriately made dimensionless — is related to the coefficient of the chiral anomaly in $4 \mathrm{D}$. In this sense, we see that there is actually a similarity in the couplings of $A_{5}$ (PNGB) and dilaton to two SM gauge bosons, i.e., both are driven by anomalies: chiral for former vs. scale anomaly for dilaton [as seen clearly in first line of eq. (2.14), i.e., the " $b_{\text {strong" }}$-form].

\section{Phenomenology}

General features. We first discuss some overall points, before studying each particle in detail. Assuming $\Lambda_{\mathrm{Higgs}} \gg \Lambda_{\mathrm{IR}}$, the couplings of the (lightest) KK/composite spin-1 gauge bosons to the SM matter (fermions and Higgs) are significant (albeit mildly suppressed relative to the SM values) and (approximately) flavor-blind: see last term in eq. (2.24) and eq. (2.32). On the other hand, radion and $\mathrm{KK} /$ composite graviton couple predominantly to pairs of SM gauge bosons and negligibly to SM matter: see eqs. (2.35), (2.38), (2.14) and (2.18). This feature is in sharp contrast to standard minimal model of figure 1, where couplings to heavy SM (top quark/Higgs/longitudinal $\mathrm{W} / Z$ ) dominate as far as decays are concerned. So, dilepton, diphoton and dijet final states are usually — and correctly — 
neglected, but now they acquire significance or even the dominant role. At the same time, the (small) flavor non-universality arising in these couplings (i.e., Higgs/top compositeness scale) can be probed by precision studies of these flavor-universal resonances (of mass $\sim \Lambda_{\mathrm{IR}}$ ), thereby distinguishing it from (purely) vector-like confinement (which corresponds to decoupling of top/Higgs compositeness scale), rather experimentally one can see the latter as a vestige of a full solution to the Planck-weak hierarchy. Finally, in the case of a unified bulk gauge symmetry, i.e., entire SM gauge group is subgroup of simple IR bulk gauge group ( $H_{\mathrm{IR}}$ of eq. (2.40)), we should of course also find that resonances come in complete degenerate unified multiplets. This is dual to the IR strong dynamics having a simple global symmetry partially gauged by SM.

\subsection{Radion/dilaton}

Dilaton production. Note that dilaton can be somewhat lighter than higher spin composites [see eq. (2.9)], thus possibly the first particle to be discovered. Rough estimates of the (total) cross-section (from gluon fusion) for $g_{\star}^{\text {grav }}=1 ; g_{\star}^{\mathrm{QCD}}=3, \Lambda_{\mathrm{IR}}=3 \mathrm{TeV}$ and $m_{\varphi}=1(2) \mathrm{TeV}$ are $\sigma_{\text {tot }} \sim 80(\sim 4.4) \mathrm{fb}^{11}$ One of these sample points was mentioned as part of table 1 in introduction.

Dilaton decay. Moving onto decays of dilaton, these are dominantly to two SM gauge bosons (based on the couplings discussed earlier, assuming $\Lambda_{\mathrm{Higgs}} \gg \Lambda_{\mathrm{IR}}$ ). It is noteworthy that in the unified case, i.e., SM gauges a subgroup of a simple global symmetry group of $4 \mathrm{D}$ strong dynamics, considering SU(5) unification as an example here, we obtain the following relation [see eq. (2.14)]:

$$
\frac{C_{\varphi \gamma \gamma}}{g_{\gamma}^{2}}: \frac{C_{\varphi Z Z}}{g_{Z}^{2}}: \frac{C_{\varphi W W}}{g_{W}^{2}}: \frac{C_{\varphi g g}}{g_{Q C D}^{2}}=\frac{8}{3}: \frac{5}{8}: 1: 1
$$

where $C_{\varphi V V}$ denotes the coupling of the dilaton to two corresponding SM gauge bosons and $g_{V}$ 's are corresponding SM gauge couplings, both being renormalized at a relevant energy scale (roughly at $\mu \sim m_{\varphi}$ ). This striking feature can be checked by measuring dilaton BR's. Numerically, BR's to $\gamma \gamma, Z Z, W W$ and $g g$ (in this unified case) are $\approx 0.7 \%$, $1 \%, 3 \%$ and $95 \%$, respectively. However, note that the above universality (among the SM gauge groups) feature applies for any $H_{\mathrm{IR}}$-singlet composite scalar. In this sense dilaton is not unique. The current bounds on cross-section $\times \mathrm{BR}$ to di-photons from resonant di-photon searches at the LHC [77, 78] are $\sim 0.5$ (0.2) fb for 1 (2) TeV mass. Similarly, di-jet searches [79] give a bound of $\sim 200 \mathrm{fb}(1 \mathrm{pb})$ for 2 (1) TeV mass. Both of these are satisfied for the above illustrative choice of parameters, although the $1 \mathrm{TeV}$ case is on the edge of the di-photon bound. Note that values of $g_{\star}^{\text {grav }}$ larger than 1 would then be ruled out (keeping other parameters the same). However, for the model with two intermediate branes to be discussed in section 4 , we will show that such values of $g_{\star}^{\text {grav }}$ can indeed satisfy the bounds.

\footnotetext{
${ }^{11}$ All cross-section numbers are for LHC13 and have been obtained using implementations of above models into Madgraph.
} 
CP structure. The CP-even structure of the couplings to SM gauge bosons for dilaton vs. CP-odd for $A_{5} /$ PNGB's (discussed above: see eqs. (2.41) and (2.14)) is an important issue. It can be discriminated by (for example) decays to $Z Z \rightarrow$ four leptons, using the additional observables therein, i.e., corresponding to polarization of $Z$ (as compared to using just angular distribution of spin-summed SM gauge boson taken as "final" state, which is the same for both cases) [80].

\section{$3.2 \quad$ Spin-1 composite}

Here, we have more than one type, each with several competing decay channels. So, we need more detailed analysis for obtaining bounds/signals. We give some general arguments first. In the unified case, based on same mass and composite coupling as in eq. (2.24), we should find for $\mathrm{SU}(5)$ unification as an example (similarly to the radion above)

$$
\frac{\sigma_{q \bar{q} \rightarrow \rho_{\gamma}}}{g_{\gamma}^{4}}: \frac{\sigma_{q \bar{q} \rightarrow \rho_{Z}}}{g_{Z}^{4}}: \frac{\sigma_{q \bar{q} \rightarrow \rho_{W}}}{g_{W}^{4}}: \frac{\sigma_{q \bar{q} \rightarrow \rho_{g}}}{g_{Q C D}^{4}}=\frac{8}{3}: \frac{5}{8}: 1: 1
$$

where $\sigma_{q \bar{q} \rightarrow \rho_{V}}$ denotes the production cross section of the composite spin-1 resonance which mixes with external gauge boson $\mathrm{V}$ and $g_{V}$ 's are corresponding SM gauge couplings renormalized at a relevant energy scale (roughly at $\mu \sim M_{\text {KKgauge }}$ ). In the non-unified case, while the above relations do not apply, the following correlation between radion decays and spin-1 production cross-section can nonetheless be tested: as seen from eqs. (2.14) and (2.24), we expect

$$
\begin{aligned}
\frac{\text { coupling of dilaton to SM gauge boson } \times(\text { gauge coupling })^{2}}{(\text { corresponding }) \text { composite spin-1 cross-section }} & \approx \text { same for all SM gauge groups } \\
& \propto g_{\star}^{\text {grav }} .
\end{aligned}
$$

Remarkably, in spite of apparent lack of unification (i.e., $b_{\text {strong }}$ is different for different gauge groups), we find that the above ratio is universal! Moreover, it applies only for the case of composite scalar being dilaton, i.e., the above relation is not valid for a generic scalar composite. In contrast, in the unified case, the above correlation is not independent of the two separate relations discussed earlier, i.e., eqs. (3.1) and (3.2).

Note that the universal constant on r.h.s. of eq. (3.3) involves $g_{\star}^{\text {grav }}$ [apart from other known factors: see eq. (2.14) and last term of eq. (2.24)]. Thus, independent determination of $g_{\star}^{\text {grav }}$, for example, from KK graviton measurements could provide an interesting test of this framework using eq. (3.3). This would apply to both unified and non-unified cases discussed above.

\subsubsection{Current bounds in flavor-universal limit}

Based on the suppressed (as compared to the SM, but still non-negligible) and flavoruniversal coupling in the last term of eq. (2.24), we find that spin-1 masses of a few $\mathrm{TeV}$ are still consistent with the LHC searches performed so far in multiple channels. We now move onto more details, discussing bounds on KK $Z$ first, followed by KK gluon. 


\section{KK $Z$ :}

(i) Di-lepton: note that composite/KK $Z$ in this case is (approximately) like sequential SM $Z^{\prime}$, but with coupling to light quarks inside proton (the dominant production mechanism) being reduced by $\sim g_{\mathrm{EW}} / g_{\star}^{\mathrm{EW}}$. We find that predicted cross-section of sequential SM $Z^{\prime}$ exceeds the bound [81, 82] by $\sim 70(25)$ for $M_{Z^{\prime}} \sim 2(2.5) \mathrm{TeV}$. Translating this bound to our case, we get (setting $g_{\mathrm{EW}} \sim 0.6$ ):

$$
\begin{aligned}
\Lambda_{\mathrm{IR}} & \gtrsim 2 \mathrm{TeV} \text { for } g_{\star}^{\mathrm{EW}} \sim 5 \\
& \gtrsim 2.5 \mathrm{TeV} \text { for } g_{\star}^{\mathrm{EW}} \sim 3
\end{aligned}
$$

Of course, only the smaller values of $g_{\star}^{\text {gauge }}(\sim 3)$ are compatible with a controlled $5 \mathrm{D}$ description, but the somewhat larger values $(\sim 5)$ are still reasonable from the viewpoint of (purely) 4D strong dynamics, for example, $\rho \pi \pi$ coupling in real-world QCD is roughly of this size. We can of course interpolate for other composite spin-1 masses. To be more precise, we will have to add bound from composite photon (above was just composite $Z$ ) but as an estimate what we did should suffice. Similarly, we can obtain a bound on KK $W$ in our model based on the searches for $W^{\prime}$ 's (via their leptonic decays) at the LHC [83, 84]: we find that it is (roughly) comparable to that on the KK Z and KK gluon (as we discuss below).

(ii) Di-boson: even in the flavor-universal limit $\left(\Lambda_{\text {Higgs }} \rightarrow \infty\right) \mathrm{KK} Z / W$ couples also to Higgs (including longitudinal $W / Z$, i.e., "di-bosons"). So, we can rescale from bound for heavy vector triplet (HVT) model [85], which is (roughly) similar to standard warped/composite case of figure 1 (i.e., couplings to Higgs/top dominate): the current bound $[86,87]$ on the mass is $2.8 \mathrm{TeV}$ for $g_{\star}^{\mathrm{EW}}=3$. However, composite $W / Z$ 's for the above HVT model decay to dibosons with a BR of $\approx 100 \%$, since couplings to dibosons are (much) larger than to the SM fermions, latter being assumed to be flavor-universal. On the other hand, in the (fully) flavor-universal limit that we are considering here, we can readily estimate that the BR to dibosons is reduced to (roughly) $4 \%$, in which case, bound is weaker than $2 \mathrm{TeV}$ (rescaling from the experimental plots).

So, we conclude that di-lepton bound for our KK $Z$ case is a bit stronger than di-boson. Just for completeness' sake, we mention that there is also a $Z^{\prime}$ bound of $2-2.5 \mathrm{TeV}$ from the di-jet search [79]. However, this assumes coupling to light quarks inside proton is same as SM $Z$, vs. smaller here. Similarly, $Z^{\prime}$ bound from di-top is $\sim 2.5 \mathrm{TeV}[88,89]$, but that is for a model with enhanced (even with respect to the SM) coupling to first and third generations [90]; hence for our case, bound should be weaker. Overall, then di-jet and d-top bounds for KK $Z$ are sub-dominant to that from di-lepton discussed earlier.

\section{KK gluon:}

(i) Di-top: similarly to KK $Z / W$ above, we can rescale from the KK gluon bounds [88, 89]: the predicted cross-section [all for $g_{\star}^{\mathrm{QCD}} \sim 5$, as assumed in [91], which is quoted 
in $[88,89]]$ is larger than bound by $\sim 6(2)$ for mass of KK gluon of $2(2.5) \mathrm{TeV}$. The above bounds are assuming $\mathrm{BR}$ to top quarks $\approx 1$ (as in the standard scenario) so that for our case (i.e., with BR to top quarks of $\approx 1 / 6$ instead), we get

$$
\begin{aligned}
\Lambda_{\mathrm{IR}} & \gtrsim 2.0 \mathrm{TeV} \text { for } g_{\star}^{\mathrm{QCD}} \sim 5 \\
& \gtrsim 2.5 \mathrm{TeV} \text { for } g_{\star}^{\mathrm{QCD}} \sim 3
\end{aligned}
$$

As usual, we can interpolate for other composite spin-1 masses.

(ii) Di-jet: here, we can re-scale from axigluon bounds [79], i.e., coupling to our composite gluon is smaller by a factor of $\sim g_{\mathrm{QCD}} /\left(g_{\star}^{\mathrm{QCD}} \times \sqrt{2}\right)$, since coupling of axigluon [see discussion in [92] referred to by [79]] is larger than QCD by $\sqrt{2}$. The cross-section is constrained to be smaller than the prediction for axigluon by $\sim 50$ (30) for axigluon mass of 2 (2.5) TeV. So, using the above couplings, we get for our case:

$$
\begin{aligned}
\Lambda_{\mathrm{IR}} & \gtrsim 2.0 \mathrm{TeV} \text { for } g_{\star}^{\mathrm{QCD}} \sim 5 \\
& \gtrsim 2.5 \mathrm{TeV} \text { for } g_{\star}^{\mathrm{QCD}} \sim 4
\end{aligned}
$$

Similarly, we can find the bound for other values of $\Lambda_{\mathrm{IR}}$.

So, di-top and di-jet bound are (roughly) comparable in the case of KK gluon.

\subsubsection{Probing top/Higgs compositeness}

Next, we discuss the possibility of being able to see some remnants of top/Higgs compositeness in the properties of composite resonances at $\Lambda_{\mathrm{IR}}$.

Summary. As seen from eqs. (2.32), (2.38) and (2.18), spin-1 couplings (cf. dilaton and spin-2) at the LHC are most sensitive to flavor non-universal corrections. In particular, for spin-1 composite, the net coupling [combining eqs. (2.24) and (2.32)] to SM fermions is then given (schematically) by:

$$
\delta \mathcal{L} \sim\left[-\frac{g_{\mathrm{SM}}^{2}}{g_{\star \mathrm{IR}}^{\text {gauge }}}+h \frac{g_{\star \mathrm{UV}}^{\text {gauge } 2}}{g_{\star \mathrm{IR}}^{\text {gauge }}}\left(\frac{\Lambda_{\mathrm{IR}}}{\Lambda_{\mathrm{Higgs}}}\right)^{2}\right] \bar{q} \gamma^{\mu} \rho_{\mu} q
$$

Here, $h$ is an $O(1)$ factor which depends on details of the model (whether a $4 \mathrm{D}$ composite theory or $5 \mathrm{D}$ dual). Note that the $5 \mathrm{D}$ model gives opposite sign for the flavor non-universal coupling (to top/Higgs) of spin-1 vs. flavor universal one, i.e., $h>0$, whereas from purely $4 \mathrm{D}$ CFT viewpoint, $h<0$ cannot be ruled out. Eq. (3.10) shows that the non-universal contributions (second term above) start becoming relevant (i.e., comparable to the universal first term) for:

$$
\frac{\Lambda_{\mathrm{Higgs}}}{\Lambda_{\mathrm{IR}}} \sim \frac{g_{\star \mathrm{UV}}^{\text {gauge }}}{g_{\mathrm{SM}}}
$$

Setting $\Lambda_{\mathrm{IR}} \sim 3 \mathrm{TeV}$; a universal $g_{\star \mathrm{UV}}^{\text {gauge }} \sim 3 ; g_{\mathrm{EW}} \sim 0.6$ and $g_{\mathrm{QCD}} \sim 1$, we see that above equality occurs (roughly) for

$$
\Lambda_{\text {Higgs }} \sim 10(15) \mathrm{TeV} \text { for KK gluon }(Z)
$$


which is (roughly) the flavor bound, i.e., (in general) we do expect sensitivity to top/Higgs compositeness! Again, note that in the standard scenario, i.e., $\Lambda_{\text {Higgs }} \sim \Lambda_{\mathrm{IR}}$, the nonuniversal contribution actually dominates: see eq. (3.10).

KK gluon vs. KK $Z$. In particular, KK gluon might be especially promising in this regard, since for the flavor-universal case, di-jet bounds on KK gluon seem comparable to di-top as indicated above, which suggests that there should be significant sensitivity to above perturbations, for example, non-universal coupling to top being comparable to universal might then show up even at discovery stage! Whereas, in flavor-universal limit, it seems bounds from di-boson/di-top are somewhat weaker than from di-lepton final state for $\mathrm{KK} Z$, thus suggesting that probe of top/Higgs compositeness (again, for the case when flavor non-universal couplings are comparable to flavor universal ones) might have to wait for post-discovery precision-level studies. On the other hand, as discussed above, for the same top/Higgs compositeness scale, flavor non-universal effects are actually a bit larger for KK $Z$ than for KK gluon. So, overall, the two modes might be complementary in this regard.

Details of analysis. Estimates of various BR's illustrating the above ideas are given in table 2: these were already mentioned in the introduction, including the tables. We now present more details. First, as a reminder, in this table 2, we fix $\mathrm{KK} Z /$ gluon mass to be $3 \mathrm{TeV}$ and the composite gauge coupling $\left(g_{\star \mathrm{UV}}^{\text {gauge }}\right)$ to be 3 . Hence, the production cross-section is the same throughout the tables, but we vary Higgs compositeness scale.

These numbers are obtained simply using the net coupling given in eq. (3.10). Just for the sake of concreteness, we choose a "central" value for the $O(1)$ coefficient $h$ in eq. (3.10) so that $\Lambda_{\mathrm{Higgs}}=10$ and $15 \mathrm{TeV}$ gives exact equality between the two terms there for $\mathrm{KK}$ gluon and KK $Z$, respectively. Then, for each $\Lambda_{\text {Higgs }}$, we vary $h$ between a factor of 2 and $1 / 2$ around this central value. Thus, we obtain a range of BR's even for fixed $\Lambda_{\text {Higgs. }}$. Mostly for simplicity, we assume only $t_{R}$ (and Higgs) is (fully) composite, i.e., $(t, b)_{L}$ 's compositeness is smaller. Also, we will assume of $h>0$ (based on 5D model, as mentioned above). We then see that for values of $\Lambda_{\text {Higgs }} / \Lambda_{\text {IR }}$ around eq. (3.11), there is actually a possibility of "cancellation" between the two terms in eq. (3.10); this feature is reflected in these tables in BR's to top/dibosons becoming smaller than flavor-universal limit as we start lowering the Higgs compositeness scale from a high value. Note that, as reflected by our $O(1)$ variation of $h$ factor, we are not really contemplating a fine-tuning here, rather only pointing out that a mild suppression is possible in this way. Eventually, i.e., for even lower $\Lambda_{\text {Higgs }}$, of course the non-universal part of couplings to top/Higgs dominates over universal one so that BR's to top/Higgs become larger, as they asymptote to the values of the minimal model of figure 1.

Finally, we have to consider the decay of (composite) spin-1 to a dilaton and a SM gauge boson. Based on eqs. (2.14) and (2.30), it is straightforward to show that there exists choices of the relevant parameters such that this decay is (much) smaller than to the SM fermions. For simplicity, here we assume that is the case in tables shown above. Having said this, a dilaton and a SM gauge boson is an interesting final state (followed by dilation $\rightarrow$ two SM gauge bosons), which (to the best of our knowledge) has not been 
studied before. In fact, in ongoing work, we are determining (other) regions of parameter space where this new decay channel actually dominates over the SM fermion pair mode and analyzing the corresponding LHC signals. Also, in this case, the BR to SM fermion pairs is suppressed, thereby relaxing the bound on gauge KK particles that were discussed earlier.

As anticipated earlier (but now seen more explicitly in the tables), as we lower Higgs compositeness scale from decoupling limit, at $\sim O(10) \mathrm{TeV}$, we start seeing $\sim O(1)$ deviations from flavor-blindness (middle vs. rightmost columns), that too "earlier" for KK $Z$ than for KK gluon. At the same time, these BR's significantly different than standard Higgs compositeness case (leftmost column). So, the moral here is that composite $Z$ /gluon can provide "glimpse" into Higgs/top compositeness, provided that this scale is not too far from the lower limit from flavor/CP violation, i.e., $\sim O(10) \mathrm{TeV}$.

Other values of KK masses. For the sake of completeness, we mention that the (total) cross-sections for 2 and $4 \mathrm{TeV}$ composite/KK $Z$ and gluon for $g_{\star \mathrm{IR}}^{\text {gauge }}=3$ (at $\sqrt{s}=13 \mathrm{TeV}$ LHC) are $\sim 28,0.3 \mathrm{fb}(Z)$ and $\sim 1834,17 \mathrm{fb}$ (gluon), respectively (of course, the $2 \mathrm{TeV}$ case might be ruled out as per above discussion, unless we invoke extra decay modes, for example to light $A_{5}$ 's). From eq. (3.11), it is clear that as we vary composite spin-1 masses in this way, one could then be sensitive to lower/higher top/Higgs compositeness scale.

Comparison to other probes of top/Higgs compositeness. Let us summarize by comparing the above signals of top/Higgs compositeness scale of $O(10) \mathrm{TeV}$ to other approaches. One of the standard probes would be existing/upcoming low-energy flavor experiments, which will be sensitive to $\Lambda_{\text {Higgs }} \sim O(10)$ almost by construction, since $O(10) \mathrm{TeV}$ was chosen to barely satisfy the current flavor/CP violation bounds. Of course, this would provide the most indirect view, for example, even if we see a signal, we cannot be sure about which underlying new physics it corresponds to, i.e., whether it is $\Lambda_{\text {Higgs }} \sim O(10) \mathrm{TeV}$ of the warped/composite Higgs framework or some thing else. On the other hand, the most direct signal is possible at a future $100 \mathrm{TeV}$ hadron collider, where the associated, i.e., $O(10) \mathrm{TeV}$, physics of compositeness can be produced without any suppression. In fact, this could serve as a motivation to build such a machine.

Here, we showed how extending the usual, minimal framework to include a intermediate brane (figure 3) results in novel probe of the general framework. Namely, it creates a new threshold, i.e., a few $\mathrm{TeV}$ resonances intermediate in mass between $O(10)$ flavor scale and the SM/weak scale itself, whose leading couplings are flavor-universal, rendering such a mass scale safe from flavor bounds. This angle actually combines some of the virtues of both the above approaches, for example, we can directly produce the relevant particles at the ongoing LHC. Of course, simply discovering these few-TeV particles in flavor-blind channels - even if very exciting! — would not quite constitute a smoking-gun of top/Higgs compositeness which lies at the core of this framework. Remarkably, we have seen above that the non-universal contributions to the couplings of these few $\mathrm{TeV}$ particles — stemming from top/Higgs compositeness - are not far behind. Hence, precision studies of these new states can indeed unravel these effects. Clearly, this sensitivity to $O(10) \mathrm{TeV}$ compositeness scale is intermediate between explicit production of compositeness physics by a $100 \mathrm{TeV}$ collider and indirect low energy flavor tests. 
Finally, we mention (other) virtual effects of this $\Lambda_{\text {Higgs }}$ physics at the LHC such as on precision Higgs or top couplings measurements or analysis of continuum top/Higgs production. However, given $\Lambda_{\text {Higgs }} \sim O(10) \mathrm{TeV}$, even the high-luminosity LHC will not be sensitive to the effects in these searches. The point is that such probes lack the resonanceenhancement $^{12}$ that the above lighter spin-1 studies afford: again, both these effects do have a (common) (few $\left.\mathrm{TeV} / \Lambda_{\text {Higgs }}\right)^{2}$ suppression.

\section{$3.3 \quad$ Spin-2 composite}

The (total) cross-sections (again, from gluon fusion, at $\sqrt{s}=13 \mathrm{TeV} \mathrm{LHC}$ ) are $\sim 40$ (1.8) fb for $\Lambda_{\mathrm{IR}}=2(3) \mathrm{TeV}$ for $g_{\star \mathrm{IR}}^{\text {grav }}=1$ and $g_{\star}^{\mathrm{QCD}}=3$. Just like for dilaton mentioned above, decays are dominated by two SM gauge bosons, unless $m_{\varphi}<\frac{1}{2} M_{\mathrm{KKgrav}}$, in which case, decay to the dilatons dominates (due to stronger coupling). Furthermore, in the unified case, we get coupling of spin-2 to two SM gauge bosons $\propto$ corresponding (SM gauge coupling) ${ }^{2}$. Thus, (neglecting decays to dilaton, for example, assuming $m_{\varphi}>\frac{1}{2} M_{\text {KKgrav }}$ ) BR's to $\gamma \gamma, Z Z, W W$, and $g g$ are $\approx 0.7 \%, 1 \%, 3 \%$ and $95 \%$, respectively (like for radion). It is also clear that current bounds on cross-section from resonant di-photon search are satisfied for above choice of parameters, since there is not much difference between spin-0 and spin-2 here in so far as experimental bounds are concerned.

Significance of spin 2. Even though the final state for composite/KK graviton might be similar to dilaton (i.e., two SM gauge bosons), obviously, spin- 2 vs. spin-0 can be distinguished using angular distributions. In fact, as already mentioned earlier, a random spin-2 has three different angular amplitudes [73] vs. KK graviton having only one (i.e., coupling to $T_{\mu \nu}$ only), hence providing disambiguation between generic strong dynamics and extra-dimensional frameworks (i.e., dual to a special structure of strong dynamics). Finally, it is interesting that mere discovery of spin-2 implies that there is an infinite tower of heavier states (whether composite or KK) because the theory of (massive) spin-2 is nonrenormalizable (vs. spin-0 or 1), thus guaranteeing more and rich discoveries in the future!

\section{Model with two intermediate branes}

Our work opens up other possibilities also: most significantly, we can have the gauge brane split (at $\left.\Lambda_{\text {meson }}\right)$ from gravity $\left(\Lambda_{\text {glueball }}\right)$ as in figure 3 . In this case, KK graviton/radion will be the lightest; in particular, radion can be lighter than KK graviton, as seen from eq. (2.9). ${ }^{13}$ So, we have (parametrically speaking) $m_{\varphi} \lesssim \Lambda_{\text {glueball }} \ll \Lambda_{\text {meson }} \ll \Lambda_{\text {Higgs. }}$. Also, stabilization of the inter-brane separations (in this case, we have three of them) can be done via a generalization of what was done for the model with one intermediate brane above.

In more detail, the couplings of KK graviton and radion to SM gauge bosons will be suppressed by $\left(\Lambda_{\text {meson }} / \Lambda_{\text {glueball }}\right)^{4}$ in this model, similarly to the case of their couplings to

\footnotetext{
${ }^{12}$ In fact, these states are quite narrow. For example, with the assumptions made above and for $\Lambda_{\text {Higgs }} \sim$ $15 \mathrm{TeV}$, we estimate that $\Gamma / M$ for $\mathrm{KK} Z$ is $O(0.1 \%)$.

${ }^{13}$ In fact, (very) recently [93] studied a $4 \mathrm{D}$ model (with new — pure glue — strong dynamics) which is sort of dual of the above gauge-gravity split case (with the lightest scalar glueball being roughly the radion/dilaton).
} 


\begin{tabular}{|c|c|c|}
\hline $\begin{array}{ll} & \text { Type of coupling } \\
\text { Resonance } & \end{array}$ & Higgs compositeness-sensitive & flavor-blind \\
\hline dilaton & $\frac{g_{x}^{\text {grav }}}{\Lambda_{\text {glueball }}}\left(\frac{\Lambda_{\text {glueball }}}{\Lambda_{\text {Higgs }}}\right)^{4}\left[m_{t} \bar{t} t+(\partial H)^{2}\right]$ & $\frac{g_{\mathrm{g}}^{\text {grav }}}{\Lambda_{\mathrm{glueball}}}\left(\frac{g_{\mathrm{SM}}}{g_{\star}^{\text {gaulge }}}\right)^{2}\left(\frac{\Lambda_{\text {glueball }}}{\Lambda_{\text {meson }}}\right)^{4} F^{\mu \nu} F_{\mu \nu}$ \\
\hline KK $Z$ & $g_{\star}^{\text {gauge }}\left(\frac{\Lambda_{\text {glueball }}}{\Lambda_{\text {Higgs }}}\right)^{2}\left(\bar{t} \gamma^{\mu} t+H^{\dagger} \partial^{\mu} H\right)$ & $\frac{g_{\mathrm{EW}}^{2}}{g_{\star}^{\text {gauge }}}\left(\bar{q} \gamma^{\mu} q+\bar{l} \gamma^{\mu} l\right)$ (all generations) \\
\hline KK gluon & $g_{\star}^{\text {gauge }}\left(\frac{\Lambda_{\text {glueball }}}{\Lambda_{\text {Higgs }}}\right)^{2} \bar{t} \gamma^{\mu} t$ & $\frac{g_{\mathrm{QCD}}^{2}}{g_{\star}^{\text {gauge }}} \bar{q} \gamma^{\mu} q($ all generations $)$ \\
\hline KK graviton & $\frac{g_{\star}^{\text {grav }}}{\Lambda_{\text {glueball }}}\left(\frac{\Lambda_{\text {glueball }}}{\Lambda_{\text {Higgs }}}\right)^{4} T_{\mu \nu}^{(t / H)}$ & $\frac{g_{\star}^{\text {grav }}}{\Lambda_{\text {glueball }}}\left(\frac{g_{\mathrm{SM}}}{g_{\star}^{\text {gauge }}}\right)^{2}\left(\frac{\Lambda_{\text {glueball }}}{\Lambda_{\text {meson }}}\right)^{4} T_{\mu \nu}^{\text {(gauge) }}$ \\
\hline
\end{tabular}

Table 4. Summary of universal and non-universal couplings of various composites in the model with two intermediate branes.

top/Higgs in the model of figure 5 studied here. As discussed in sections 2.3.1 and 2.3.2, these couplings result from exchange of (heavy) physics at $\Lambda_{\text {meson. }}$. Essentially, we perform the replacements $T_{\mu \nu}^{(t / H)} \rightarrow T_{\mu \nu}^{(\text {gauge) }}$ in eq. (2.38) and Higgs kinetic term $\rightarrow F_{\mu \nu} F^{\mu \nu}$ in eq. (2.18), along with $\Lambda_{\text {Higgs }} \rightarrow \Lambda_{\text {meson }}$ in both equations. On the other hand, couplings of dilaton/spin-2 to top/Higgs and those of spin-1 to all SM matter remain the same. Here, we simply summarize all these couplings in table 4 (cf. table 3 ).

Note that for fixed mass of the spin- 1 composites $\left(\Lambda_{\text {meson }}\right)$, the couplings of the lightest states in this model (i.e., KK graviton/radion) relevant for their production (i.e., to gluons) become weaker as we lower $\Lambda_{\text {glueball }}$, i.e., their mass. On the other hand, PDF's relevant for production are enhanced in this process, providing some compensation. Remarkably, it turns out that within the range of interest the former effect (i.e., weaker couplings) tends to dominate so that the cross-sections actually reduce (i.e., bounds and visibility get weaker) as we lower the KK graviton/radion mass.

A sample point is as follows: $\Lambda_{\text {meson }}=3 \mathrm{TeV}, m_{\varphi}=\Lambda_{\text {glueball }}=1 \mathrm{TeV}, g_{\star}^{\mathrm{QCD}}=3$ and $g_{\star \mathrm{IR}}^{\text {grav }}=3$ gives (total) cross-section of $\sim 3.9 \mathrm{fb}$ and $\sim 1 \mathrm{fb}$, respectively, for KK graviton and dilaton (former being larger mostly due to multiple polarizations). The decay BR's are similar to the model with one intermediate brane case. Note that gauge KK/spin-1 composite cross-section at this point are comparable to/larger than that of graviton/dilaton; in fact, the gauge KK would be strongly constrained (if not ruled out), assuming decays directly to SM particles (as discussed above). However, the spin-1 states can decay directly into non-SM particles such that they are effectively "hidden" from SM pair-resonance searches such as dileptons or dijets. For example, light $A_{5}$ 's (dual to PNGB's) can provide such channels. ${ }^{14}$ In this way, KK graviton/dilaton can actually be the most visible channel. Table 1 in the introduction had already displayed this interesting possibility.

Based on the discussion in section 2.3.3 of KK graviton contributions to precision tests, it is clear that the only relevant constraint on the KK graviton mass scale, i.e., $\Lambda_{\text {glueball }}$, in this model comes from direct LHC searches; in particular, using the cross-sections given above and bounds given earlier, we see that $\Lambda_{\text {glueball }}$ is then allowed to be as low as $\sim 1 \mathrm{TeV}$ (or even smaller).

\footnotetext{
${ }^{14}$ For a recent application of this idea in the context of the $750 \mathrm{GeV}$ diphoton excess at the LHC, see [59].
} 


\section{Conclusions/outlook}

The LHC Run 1 complemented by electroweak/flavor/CP precision tests have thus far seen no deviations from the SM. In light of this we must conclude that the principle of Naturalness, that predicts new physics below the TeV scale, is either (i) at the cusp of discovery at the LHC, (ii) playing itself out in some exceptional dynamics (such as Twin Higgs theory [94]) that evades our standard experimental probes, or (iii) that the principle is compromised in some way. Our efforts must be directed at all these options. Higgs compositeness (AdS/CFT dual to warped extra-dimensions) within the LHC reach remains a strongly motivated possibility for (i), but requires some new refinement of the warped GIM mechanism. This paper is directed instead to the option (iii) in the same, broad framework. Indeed, it is noteworthy that the minimal incarnation of this paradigm (see figure 1) can readily and elegantly fit the experimental facts if we take the related new physics to live at $\sim O(10) \mathrm{TeV}$, solving the "big hierarchy problem" between the electroweak and Planck scales, but leaving unexplained a "little hierarchy problem". It is not the modest associated fine-tuning that disturbs us here but the fact that the solution to the hierarchy problem would then lie out of LHC reach!

We have shown that a simple extension of the above model can also readily fit all the experimental facts if the physics of naturalness is deferred until $\sim O(10) \mathrm{TeV}$. Namely, when different fields propagate different amounts into the IR of the extra dimension (see figure 3), there can naturally be lighter $\mathrm{TeV}$-scale "vestiges" of the heavy naturalness physics within LHC reach, in the form of new spin-0,1,2 resonances, identified as KK excitations of the extra dimension or composites in the dual mechanism of vectorlike confinement. Although they would constitute a rich new physics close at hand, they escape the strong constraints from flavor/CP tests by virtue of their largely flavor-blind, gauge-mediated couplings to the standard model. We have described several striking features of their phenomenology in the 5D Randall-Sundrum framework and its AdS/CFT dual. In particular, search channels at the LHC such as dileptons, dijets and diphotons for the $\sim \mathrm{TeV}$-mass resonances acquire significance in this framework, cf. decays being dominated by top/Higgs in the minimal model of figure 1. In addition (and in contrast to the minimal model of figure 1), the radion and KK graviton (i.e., the gravitational sector) can be readily lighter than other states and, in part of the parameter space, can even lead to first discovery.

But flavor-blindness, however rich the physics, also suggests blindness to the solution to the hierarchy problem. Fortunately, we saw there are small deviations from flavorblindness in resonance decays into top/Higgs rich final states. These processes thereby give a resonance-enhanced "preview" of Higgs compositeness at the LHC, even though the Higgs compositeness scale and its ultimate resolution of the hierarchy problem is out of LHC reach! This provides a pathway in which LHC discoveries might set the stage for even higher energy explorations. A roughly comparable analogy within the supersymmetric paradigm is (mini-) Split SUSY [95, 96], in which the stops most directly relevant to the hierarchy problem lie above LHC reach (helping to explain the larger-than-expected Higgs boson mass) while spin-1/2 super-partners are significantly lighter. Seeing the lighter super-partners at the LHC with their SUSY-specific quantum numbers would also give a 
"preview" of the supersymmetric solution to the hierarchy problem, which could be fully confirmed by going to higher energy colliders.

In future work, it will be interesting to study in more detail the LHC signals for the (approximately) flavor-blind $\sim \mathrm{TeV}$ mass resonances which were outlined here, including what we can learn about the physics of top/Higgs compositeness at $\sim O(10) \mathrm{TeV}$ from their precision analysis. In addition, we plan to initiate investigation of more direct signals of the latter physics which might be possible at a future $100 \mathrm{TeV}$ hadron collider.

We are now at the beginning of LHC Run 2, and it is essential that theory lays out the most plausible and powerful mechanisms within reach. In the language of $4 \mathrm{D}$ strong dynamics we have shown that vectorlike confinement arising in the IR of Higgs compositeness is such a plausible form of new physics, already exciting at the LHC and able to pave the way for an even more ambitious program of discovery at future higherenergy colliders.

\section{Acknowledgments}

We would like to thank Zackaria Chacko for discussions. This work was supported in part by NSF Grant No. PHY-1315155 and the Maryland Center for Fundamental Physics. SH was also supported in part by a fellowship from The Kwanjeong Educational Foundation.

\section{A Details of choice of parameters}

\section{A.1 Matching at the intermediate/Higgs brane}

We assume the same 5D Planck scale $\left(M_{5}\right)$ throughout the bulk. However, in short, the bulk cosmological constant (CC) - and hence AdS curvature scale $(k)$ - will be different in the matter/Higgs and gauge/gravity (only) bulks due to presence of (tension on) the intermediate/Higgs brane. In more detail, we define

$$
\begin{aligned}
\mathrm{CC}_{\mathrm{UV}(\mathrm{IR})} & \equiv 24 M_{5}^{3} k_{\mathrm{UV}(\mathrm{IR})}^{2} \\
k_{\mathrm{UV}(\mathrm{IR})} & \equiv \frac{1}{R_{\mathrm{UV}(\mathrm{IR})}^{\mathrm{AdS}}}
\end{aligned}
$$

where "UV" and "IR" denote the bulks on the two sides of the Higgs brane and $R^{\text {AdS }}$ is the AdS curvature radius. Solving Einstein's equations across the the Higgs brane (with tension, $\left.T_{\text {Higgs }}\right)$ gives [97]:

$$
T_{\mathrm{Higgs}}=12 M_{5}^{3}\left(k_{\mathrm{IR}}-k_{\mathrm{UV}}\right)
$$

Since we require $T_{\text {Higgs }}>0$ in order to avoid a branon (brane-bending degrees of freedom, denoted by $Y$ ) ghost [98], we see that

$$
k_{\mathrm{UV}}<k_{\mathrm{IR}}
$$

i.e., curvature scale increases in the IR. Let us consider in the following how this new feature modifies the usual choice of parameters. 


\section{A.2 Implications of above matching}

Consider the gravity sector of the model first. Clearly, we then have two different $g_{\star}^{\text {grav }}$ s on the two sides of the Higgs brane:

$$
\frac{g_{\star \mathrm{IR}}^{\mathrm{grav}}}{g_{\star \mathrm{UV}}^{\text {grav }}}=\sqrt{\frac{k_{\mathrm{IR}}^{3}}{k_{\mathrm{UV}}^{3}}}
$$

As usual, we have bulk gravity becoming strongly coupled at [99]

$$
\Lambda_{\text {strong }}^{\text {grav }} \sim k\left(\frac{16 \pi^{2}}{g_{\star}^{\text {grav } 2}}\right)^{1 / 3}
$$

Suppose we would like to have at least $N_{\mathrm{KK}}^{\min }$ number of weakly-coupled KK modes (i.e., that much gap between 5D cut-off and curvature scale as our control parameter). Then we must have

$$
g_{\star}^{\mathrm{grav}} \lesssim \sqrt{\frac{16 \pi^{2}}{N_{\mathrm{KK}}^{\min 3}}}
$$

from the condition that $\Lambda_{\mathrm{strong}}^{\text {grav }} \gtrsim N_{\mathrm{KK}}^{\min } k$. Note that this is required in each of the two bulks, i.e., for both $g_{\star}^{\text {grav }}$, UV . Of course, in order to avoid large hierarchies amongst fundamental/5D parameters (for example, between $k$ and $M_{5}$ ), we would also impose that $g_{\star}^{\text {grav }}$ is not $\ll 1$.

Moving onto gauge sector, we similarly have

$$
\frac{g_{\star \mathrm{IR}}^{\text {gauge }}}{g_{\star \mathrm{UV}}^{\text {gauge }}}=\sqrt{\frac{k_{\mathrm{IR}}}{k_{\mathrm{UV}}}}
$$

The strong coupling scale is given by :

$$
\Lambda_{\text {strong }}^{\text {gauge }} \sim k \frac{16 \pi^{2}}{N_{\mathrm{SM}} 3 g_{\star}^{\text {gauge } 2}}
$$

where $N_{\mathrm{SM}}$ denotes size of the SM gauge group (take it here to be 3 for color) and factor of 3 in denominator above (i.e., enhancement of loop expansion parameter) comes from counting helicities of spin-1 field. So, the associated request (i.e, imposing $\Lambda_{\text {strong }}^{\text {gauge }} \gtrsim N_{\mathrm{KK}}^{\min } k$ ) is

$$
g_{\star}^{\text {gauge }} \lesssim \frac{4 \pi}{3 \sqrt{N_{\mathrm{KK}}^{\min }}}
$$

for each of the two bulks.

On the other hand, fitting to the observed/SM gauge coupling gives lower limits on $g_{\star}^{\text {gauge }}$ as follows (note that there is no analog of Landau pole for gravity, hence no lower limit on $g_{\star}^{\text {grav }}$ on this count). Consider the running of the SM gauge couplings from the UV cut-off to the IR shown in eq. (2.11). Plugging in the low-energy values of $g_{\mathrm{SM}}$ and $b_{\mathrm{SM}}$ into eq. (2.11), we find (assuming $\Lambda_{\mathrm{Higgs}} \sim 10 \mathrm{TeV}$ and $\Lambda_{\mathrm{IR}} \sim$ few $\mathrm{TeV}$ )

$$
g_{\star \mathrm{UV}}^{\text {gauge }} \gtrsim 3
$$


from the requirement that $1 / g_{\mathrm{UV}}^{2}>0$, i.e., Landau poles for SM gauge couplings are at/above $\sim 10^{15} \mathrm{GeV}$. However, $g_{\star \mathrm{IR}}^{\text {gauge }}$ mostly unconstrained, since it contributes over a (much) smaller hierarchy.

Finally, there is another requirement that the strong coupling scale of the $Y$ selfinteractions be (at least modestly) above the curvature scale, i.e.,

$$
\mathcal{L} \ni\left(\partial_{\mu} Y\right)^{2}+\frac{\left(\partial_{\mu} Y\right)^{4}}{T_{\text {Higgs }}}
$$

results in

$$
\Lambda_{\text {strong }}^{\text {branon }} \sim\left(16 \pi^{2} T_{\text {Higgs }}\right)^{1 / 4}
$$

So we need [as usual, imposing $\Lambda_{\mathrm{strong}}^{\mathrm{branon}} \gtrsim N_{\mathrm{KK}}^{\min } k$ and using eq. (A.2)]

$$
g_{\star}^{\text {grav IR }} \lesssim\left[\frac{192 \pi^{2}}{N_{\mathrm{KK}}^{\min } 4}\left(1-\frac{k_{\mathrm{UV}}}{k_{\mathrm{IR}}}\right)\right]^{1 / 2}
$$

We can check that the following choices of couplings barely satisfy all the above needs (including giving observable LHC signals):

$$
g_{\star \mathrm{UV}}^{\text {grav }}<g_{\star \mathrm{IR}}^{\text {grav }} \lesssim 3 \text { and } g_{\star \mathrm{UV}}^{\text {gauge }} \sim 3 ; \quad g_{\star \mathrm{IR}}^{\text {gauge } 2} \approx g_{\star \mathrm{UV}}^{\text {gauge } 2}[1+O(0.1)]
$$

for a minimal request of

$$
N_{\mathrm{KK}}^{\min } \sim 2
$$

(and corresponding to $k_{\mathrm{IR}} / k_{\mathrm{UV}} \approx 1+O(0.1)$ ).

Note that $g_{\star \mathrm{IR}}^{\text {gauge }}$ and $g_{\star \mathrm{UV}}^{\text {gauge }}$ are "forced" to be close to each other, due to a combination of perturbativity (upper bound on $g_{\star}^{\text {gauV }}$ ) and Landau pole (lower bound) constraints. One possibility to relieve this tension is to reduce the UV-IR hierarchy, for example, lower the UV scale to the flavor scale of $\sim 10^{5} \mathrm{TeV}$ [100], while keeping IR scale $\sim \mathrm{TeV}$ : from eq. (2.11), we see that $g_{\star \mathrm{UV}}^{\text {gauge }} \gtrsim 2$ might then be allowed (keeping both $g_{\star}^{\text {gauge, }}$ s at/below $\sim 3$ for perturbativity).

\section{B Two dilaton system}

Here we discuss the CFT dual of stabilization of the model with one intermediate brane studied in the main text. In short, as usual, we start with a CFT at a UV cut-off $\Lambda_{U V}$. This CFT confines, i.e., scale invariance is broken, at $\Lambda_{\text {int }}$, which is to be identified with $\Lambda_{\text {Higgs }}$, i.e., scale of the Higgs brane in the specific model, but here we would like to keep the notation more general. As already mentioned, this scale can be parametrized by the VEV of dilaton/radion field [denoted by $\Phi_{\text {int }}$ of mass dimension +1 , fluctuations around which correspond to the physical dilaton $\left.\left(\varphi_{\text {int }}\right)\right]$, i.e.,

$$
\Phi_{\text {int }} \sim \Lambda_{\text {int }}+a g_{\star}^{\text {grav }} \varphi_{\text {int }} .
$$


The departure from the standard (i.e., minimal model of figure 1) script involves the resulting (daughter) theory (i.e., below $\Lambda_{\text {int }}$ ) flowing to a new fixed point. This "IR" CFT then confines at an even lower scale $\Lambda_{\mathrm{IR}}$, corresponding to the VEV of another field, $\Phi_{\mathrm{IR}}$ (associated with a second dilaton, $\varphi_{\mathrm{IR}}$ ).

In more detail, in order to stabilize the two inter-brane separations (dual to determining the various mass scale hierarchies), we perturb the (UV) CFT by adding a single scalar operator (dual to the GW field) in the UV:

$$
\mathcal{L}\left(\Lambda_{\mathrm{UV}}\right) \ni \mathcal{L}_{\mathrm{CFT} \mathrm{UV}}+\lambda \Lambda_{\mathrm{UV}}^{\epsilon \mathrm{OV}} \mathcal{O}_{\mathrm{GW}}^{\mathrm{UV}}
$$

where scaling and naive/engineering dimension of $\mathcal{O}_{\mathrm{GW}}^{\mathrm{UV}}$ is $\left(4-\epsilon_{\mathrm{UV}}\right)$ (i.e., $\lambda$ above is dimensionless). As usual, we assume that there is only one scalar operator with scaling dimension close to 4 , rest of them being irrelevant (hence being dropped from the Lagrangian). We flow to $\Lambda_{\text {int }}$ (as usual, promoting appropriately $\Lambda$ 's to $\Phi$ 's throughout):

$$
\begin{aligned}
\mathcal{L}\left(\Lambda_{\mathrm{int}}\right) \ni & \mathcal{L}_{\mathrm{CFT} \text { IR }}+\left(\partial_{\mu} \Phi_{\mathrm{int}}\right)^{2}+\lambda^{\prime} \Phi_{\mathrm{int}}^{4}+d_{1} \lambda \Phi_{\mathrm{int}}^{4}\left(\frac{\Phi_{\mathrm{int}}}{\Lambda_{\mathrm{UV}}}\right)^{-\epsilon_{\mathrm{UV}}} \\
& +\left[d_{2} \lambda\left(\frac{\Phi_{\mathrm{int}}}{\Lambda_{\mathrm{UV}}}\right)^{-\epsilon_{\mathrm{UV}}}+\tilde{\lambda}\right] \Phi_{\mathrm{int}}^{\epsilon_{\mathrm{IR}}} \mathcal{O}_{\mathrm{GW}}^{\mathrm{IR}}
\end{aligned}
$$

where $d_{1,2}$ are $O(1)$ factors.

Let us elaborate on the various terms above. The first three terms above (in first line) are as discussed earlier (i.e., for the usual minimal model). Whereas, the first new term (in second line above) comes from using the interpolation:

$$
\mathcal{O}_{\mathrm{GW}}^{\mathrm{UV}}\left(\Lambda_{\mathrm{int}}\right) \sim \mathcal{O}_{\mathrm{GW}}^{\mathrm{IR}} \Phi_{\mathrm{int}}^{\epsilon_{\mathrm{IR}}-\epsilon_{\mathrm{UV}}}+\ldots
$$

in the RG evolved explicit conformal symmetry breaking term in eq. (B.2). Here, (with obvious choice of notation) $\mathcal{O}_{\mathrm{GW}}^{\mathrm{IR}}$ is an operator of the $I R$ CFT of scaling dimension $\left(4-\epsilon_{\mathrm{IR}}\right)$ : again, we assume that there exists only one such operator. On the other hand, the second term in second line of eq. (B.3) arises from spontaneous conformal symmetry breaking at the scale $\Lambda_{\text {int }}$, i.e., even if $\lambda O_{\mathrm{GW}}^{\mathrm{UV}}$ were "absent". Given above assumption about scaling dimensions of scalar operators of the IR CFT, it is clear that both terms in second line above must involve the same operator (as the leading term), i.e., coupling of $\Phi_{\text {int }}$ to other scalar operators of the IR CFT will be irrelevant.

Finally, i.e., RG flowing to the far IR scale of $\Lambda_{\mathrm{IR}}$ and adding (for a second time) the usual term consistent with the (IR) conformal symmetry, we obtain the complete potential for the two scalar fields ( $\Phi$ 's):

$$
\begin{aligned}
\mathcal{L}\left(\Lambda_{\mathrm{IR}}\right) \ni & \left(\partial_{\mu} \Phi_{\mathrm{int}}\right)^{2}+\lambda^{\prime} \Phi_{\mathrm{int}}^{4}+d_{1} \lambda \Phi_{\mathrm{int}}^{4}\left(\frac{\Phi_{\mathrm{int}}}{\Lambda_{\mathrm{UV}}}\right)^{-\epsilon_{\mathrm{UV}}}+ \\
& \left(\partial_{\mu} \Phi_{\mathrm{IR}}\right)^{2}+\tilde{\lambda}^{\prime} \Phi_{\mathrm{IR}}^{4}+\left[d_{3} \lambda\left(\frac{\Phi_{\mathrm{int}}}{\Lambda_{\mathrm{UV}}}\right)^{-\epsilon_{\mathrm{UV}}}+d_{4} \tilde{\lambda}\right]\left(\frac{\Phi_{\mathrm{IR}}}{\Phi_{\mathrm{int}}}\right)^{-\epsilon_{\mathrm{IR}}} \Phi_{\mathrm{IR}}^{4}
\end{aligned}
$$

We have to minimize the above potential in order to determine the scales $\Lambda_{\text {int }}$ and $\Lambda_{\mathrm{IR}}$ in terms of $\Lambda_{\mathrm{UV}}$ and the scaling dimensions [we can assume that the various $\lambda$ 's are $O(1)$ ]. 
As usual, we assume $\epsilon_{\mathrm{UV}}$, IR are modestly smaller than 1. In this case, we can proceed with the minimization in steps as follows. At "leading-order" (LO), it is reasonable to assume that $\left\langle\Phi_{\text {int }}\right\rangle \sim \Lambda_{\text {int }}$ is mostly determined (as in the minimal two brane case) by first line of eq. (B.5) (i.e., potential for $\Phi_{\text {int }}$ by itself) to be:

$$
\Lambda_{\mathrm{int}} \sim\left(-d_{1} \frac{\lambda}{\lambda^{\prime}}\right)^{1 / \epsilon_{\mathrm{UV}}} \Lambda_{\mathrm{UV}}
$$

with

$$
m_{\varphi_{\text {int }}}^{2} \sim \epsilon_{\mathrm{UV}} \Lambda_{\text {int }}^{2} .
$$

Similarly, plugging $\Phi_{\text {int }}=\Lambda_{\text {int }}$ (i.e., a fixed value) into second line of eq. (B.5), i.e., effective potential for $\Phi_{\mathrm{IR}}$, will give (again, as usual):

$$
\Lambda_{\mathrm{IR}} \sim\left(-\frac{1}{\tilde{\lambda}^{\prime}}\left(d_{4} \tilde{\lambda}-\frac{d_{3}}{d_{1}} \lambda^{\prime}\right)\right)^{1 / \epsilon_{\mathrm{IR}}} \Lambda_{\mathrm{int}}
$$

with

$$
m_{\varphi_{\mathrm{IR}}}^{2} \sim \epsilon_{\mathrm{IR}} \Lambda_{\mathrm{IR}}^{2}
$$

As a (partial) consistency check of the above procedure (for obtaining the values of VEV's), we can consider the mixing (if you will, the NLO) term involving both the dilatons arising from the last two terms of second line of eq. (B.5), where $\Phi_{\text {int }}$ can be thought of as fluctuations around $\Lambda_{\text {int }}$ :

$$
\Delta m_{\varphi_{\mathrm{int}} \varphi_{\mathrm{IR}}}^{2} \sim O\left(\epsilon_{\mathrm{IR}}, \epsilon_{\mathrm{UV}}\right) \frac{\Lambda_{\mathrm{IR}}^{3}}{\Lambda_{\mathrm{int}}}
$$

We see that this results in a mixing angle between two dilatons of $\sim \epsilon\left(\Lambda_{\mathrm{IR}} / \Lambda_{\mathrm{int}}\right)^{3}$, i.e., small enough. As a further check, we can show that the first derivatives of the full potential in eq. (B.5) at above values of VEV's vanish, up to terms suppressed by (powers of) $\Lambda_{\text {IR }} / \Lambda_{\text {int }}$, i.e., the actual VEV's are close enough to those obtained by the above "piecewise" minimization of the potential. Hence, to a good approximation, we can "decouple" the two dilaton systems (as already assumed in the main text).

Open Access. This article is distributed under the terms of the Creative Commons Attribution License (CC-BY 4.0), which permits any use, distribution and reproduction in any medium, provided the original author(s) and source are credited.

\section{References}

[1] G. Panico and A. Wulzer, The composite Nambu-Goldstone Higgs, Lect. Notes Phys. 913 (2016) 1 [arXiv:1506.01961].

[2] N. Arkani-Hamed, M. Porrati and L. Randall, Holography and phenomenology, JHEP 08 (2001) 017 [hep-th/0012148] [INSPIRE]. 
[3] R. Rattazzi and A. Zaffaroni, Comments on the holographic picture of the Randall-Sundrum model, JHEP 04 (2001) 021 [hep-th/0012248] [INSPIRE].

[4] R. Contino and A. Pomarol, Holography for fermions, JHEP 11 (2004) 058 [hep-th/0406257] [INSPIRE].

[5] R. Contino, Y. Nomura and A. Pomarol, Higgs as a holographic pseudoGoldstone boson, Nucl. Phys. B 671 (2003) 148 [hep-ph/0306259] [INSPIRE].

[6] K. Agashe, R. Contino and A. Pomarol, The minimal composite Higgs model, Nucl. Phys. B 719 (2005) 165 [hep-ph/0412089] [INSPIRE].

[7] L. Randall and R. Sundrum, A large mass hierarchy from a small extra dimension, Phys. Rev. Lett. 83 (1999) 3370 [hep-ph/9905221] [INSPIRE].

[8] L. Randall and R. Sundrum, An alternative to compactification, Phys. Rev. Lett. 83 (1999) 4690 [hep-th/9906064] [INSPIRE].

[9] O. Aharony, S.S. Gubser, J.M. Maldacena, H. Ooguri and Y. Oz, Large-N field theories, string theory and gravity, Phys. Rept. 323 (2000) 183 [hep-th/9905111] [INSPIRE].

[10] M.J. Strassler, Nonsupersymmetric theories with light scalar fields and large hierarchies, hep-th/0309122 [INSPIRE].

[11] S. Kachru, D. Simic and S.P. Trivedi, Stable non-supersymmetric throats in string theory, JHEP 05 (2010) 067 [arXiv:0905.2970] [INSPIRE].

[12] T. Gherghetta, Les Houches lectures on warped models and holography, hep-ph/0601213 [INSPIRE].

[13] H. Davoudiasl, S. Gopalakrishna, E. Ponton and J. Santiago, Warped 5-dimensional models: phenomenological status and experimental prospects, New J. Phys. 12 (2010) 075011 [arXiv:0908.1968] [INSPIRE].

[14] C. Csáki, J. Hubisz and P. Meade, TASI lectures on electroweak symmetry breaking from extra dimensions, hep-ph/0510275 [INSPIRE].

[15] R. Sundrum, TASI 2004 lectures: to the fifth dimension and back, hep-th/0508134 [INSPIRE].

[16] E. Ponton, TASI 2011: four lectures on TeV scale extra dimensions, arXiv:1207.3827 [INSPIRE].

[17] D.B. Kaplan, Flavor at SSC energies: a new mechanism for dynamically generated fermion masses, Nucl. Phys. B 365 (1991) 259 [INSPIRE].

[18] K. Agashe et al., Warped extra dimensional benchmarks for Snowmass 2013, arXiv: 1309.7847 [INSPIRE].

[19] K. Agashe, A. Delgado, M.J. May and R. Sundrum, RS1, custodial isospin and precision tests, JHEP 08 (2003) 050 [hep-ph/0308036] [INSPIRE].

[20] K. Agashe, R. Contino, L. Da Rold and A. Pomarol, A custodial symmetry for Zbb, Phys. Lett. B 641 (2006) 62 [hep-ph/0605341] [INSPIRE].

[21] M. Carena, E. Ponton, J. Santiago and C.E.M. Wagner, Light Kaluza-Klein states in Randall-Sundrum models with custodial SU(2), Nucl. Phys. B 759 (2006) 202 [hep-ph/0607106] [INSPIRE]. 
[22] M. Carena, E. Pontón, J. Santiago and C.E.M. Wagner, Electroweak constraints on warped models with custodial symmetry, Phys. Rev. D 76 (2007) 035006 [hep-ph/0701055] [INSPIRE].

[23] C. Delaunay, O. Gedalia, S.J. Lee, G. Perez and E. Ponton, Ultra visible warped model from flavor triviality and improved naturalness, Phys. Rev. D 83 (2011) 115003 [arXiv: 1007.0243] [INSPIRE].

[24] T. Gherghetta and A. Pomarol, Bulk fields and supersymmetry in a slice of AdS, Nucl. Phys. B 586 (2000) 141 [hep-ph/0003129] [INSPIRE].

[25] S.J. Huber and Q. Shafi, Fermion masses, mixings and proton decay in a Randall-Sundrum model, Phys. Lett. B 498 (2001) 256 [hep-ph/0010195] [INSPIRE].

[26] S.J. Huber, Flavor violation and warped geometry, Nucl. Phys. B 666 (2003) 269 [hep-ph/0303183] [INSPIRE].

[27] K. Agashe, G. Perez and A. Soni, Flavor structure of warped extra dimension models, Phys. Rev. D 71 (2005) 016002 [hep-ph/0408134] [INSPIRE].

[28] C. Csáki, A. Falkowski and A. Weiler, The flavor of the composite pseudo-Goldstone Higgs, JHEP 09 (2008) 008 [arXiv:0804.1954] [INSPIRE].

[29] M. Blanke, A.J. Buras, B. Duling, S. Gori and A. Weiler, $\Delta F=2$ observables and fine-tuning in a warped extra dimension with custodial protection, JHEP 03 (2009) 001 [arXiv:0809.1073] [INSPIRE].

[30] M. Bauer, S. Casagrande, U. Haisch and M. Neubert, Flavor physics in the Randall-Sundrum model: II. Tree-level weak-interaction processes, JHEP 09 (2010) 017 [arXiv: 0912.1625] [INSPIRE].

[31] B. Keren-Zur et al., On partial compositeness and the CP asymmetry in charm decays, Nucl. Phys. B 867 (2013) 394 [arXiv:1205.5803] [INSPIRE].

[32] A.L. Fitzpatrick, G. Perez and L. Randall, Flavor anarchy in a Randall-Sundrum model with 5 D minimal flavor violation and a low Kaluza-Klein scale, Phys. Rev. Lett. 100 (2008) 171604 [arXiv:0710.1869] [INSPIRE].

[33] C. Csáki, A. Falkowski and A. Weiler, A Simple Flavor Protection for RS, Phys. Rev. D 80 (2009) 016001 [arXiv:0806.3757] [INSPIRE].

[34] M. Redi and A. Weiler, Flavor and CP invariant composite Higgs models, JHEP 11 (2011) 108 [arXiv: 1106.6357] [INSPIRE].

[35] R. Barbieri, D. Buttazzo, F. Sala, D.M. Straub and A. Tesi, A 125 GeV composite Higgs boson versus flavour and electroweak precision tests, JHEP 05 (2013) 069 [arXiv:1211.5085] [INSPIRE].

[36] D.M. Straub, Anatomy of flavour-changing $Z$ couplings in models with partial compositeness, JHEP 08 (2013) 108 [arXiv:1302.4651] [INSPIRE].

[37] M. König, M. Neubert and D.M. Straub, Dipole operator constraints on composite Higgs models, Eur. Phys. J. C 74 (2014) 2945 [arXiv:1403.2756] [INSPIRE].

[38] C. Csáki and L. Randall, A diphoton resonance from bulk RS, JHEP 07 (2016) 061 [arXiv: 1603.07303] [INSPIRE].

[39] ATLAS collaboration, Search for resonances in diphoton events at $\sqrt{s}=13 \mathrm{TeV}$ with the ATLAS detector, JHEP 09 (2016) 001 [arXiv: 1606.03833] [INSPIRE]. 
[40] CMS collaboration, Search for resonant production of high-mass photon pairs in proton-proton collisions at $\sqrt{s}=8$ and 13 TeV, Phys. Rev. Lett. 117 (2016) 051802 [arXiv: 1606. 04093] [INSPIRE].

[41] L. Vecchi, A flavor sector for the composite Higgs, Phys. Lett. B 727 (2013) 130 [arXiv:1206.4701] [INSPIRE].

[42] G. Panico and A. Pomarol, Flavor hierarchies from dynamical scales, JHEP 07 (2016) 097 [arXiv:1603.06609] [INSPIRE].

[43] A. Carmona, E. Ponton and J. Santiago, Phenomenology of non-custodial warped models, JHEP 10 (2011) 137 [arXiv:1107.1500] [INSPIRE].

[44] C. Kilic, T. Okui and R. Sundrum, Colored resonances at the Tevatron: phenomenology and discovery potential in multijets, JHEP 07 (2008) 038 [arXiv: 0802.2568] [INSPIRE].

[45] C. Kilic, T. Okui and R. Sundrum, Vectorlike confinement at the LHC, JHEP 02 (2010) 018 [arXiv:0906.0577] [INSPIRE].

[46] Y. Bai and R.J. Hill, Weakly interacting stable pions, Phys. Rev. D 82 (2010) 111701 [arXiv: 1005.0008] [INSPIRE].

[47] W.D. Goldberger and M.B. Wise, Modulus stabilization with bulk fields, Phys. Rev. Lett. 83 (1999) 4922 [hep-ph/9907447] [INSPIRE].

[48] S. Raby, S. Dimopoulos and L. Susskind, Tumbling Gauge Theories, Nucl. Phys. B 169 (1980) 373 [INSPIRE].

[49] I.R. Klebanov and M.J. Strassler, Supergravity and a confining gauge theory: duality cascades and $\chi_{S B}$ resolution of naked singularities, JHEP 08 (2000) 052 [hep-th/0007191] [INSPIRE].

[50] R. Franceschini et al., What is the $\gamma \gamma$ resonance at 750 GeV?, JHEP 03 (2016) 144 [arXiv: 1512.04933] [INSPIRE].

[51] Y. Nakai, R. Sato and K. Tobioka, Footprints of new strong dynamics via anomaly and the $750 \mathrm{GeV}$ diphoton, Phys. Rev. Lett. 116 (2016) 151802 [arXiv: 1512.04924] [InSPIRE].

[52] M. Low, A. Tesi and L.-T. Wang, A pseudoscalar decaying to photon pairs in the early LHC Run 2 data, JHEP 03 (2016) 108 [arXiv: 1512.05328] [INSPIRE].

[53] K. Harigaya and Y. Nomura, Composite models for the $750 \mathrm{GeV}$ diphoton excess, Phys. Lett. B 754 (2016) 151 [arXiv: 1512.04850] [INSPIRE].

[54] E. Molinaro, F. Sannino and N. Vignaroli, Minimal composite dynamics versus axion origin of the diphoton excess, Mod. Phys. Lett. A 31 (2016) 1650155 [arXiv:1512.05334] [INSPIRE].

[55] B. Bellazzini, R. Franceschini, F. Sala and J. Serra, Goldstones in diphotons, JHEP 04 (2016) 072 [arXiv: 1512.05330] [INSPIRE].

[56] J. Barnard, T. Gherghetta, T.S. Ray and A. Spray, The unnatural composite Higgs, JHEP 01 (2015) 067 [arXiv:1409.7391] [INSPIRE].

[57] J. Barnard, P. Cox, T. Gherghetta and A. Spray, Long-lived, colour-triplet scalars from unnaturalness, JHEP 03 (2016) 003 [arXiv: 1510.06405] [INSPIRE].

[58] H. Davoudiasl, J.L. Hewett and T.G. Rizzo, Brane localized curvature for warped gravitons, JHEP 08 (2003) 034 [hep-ph/0305086] [INSPIRE]. 
[59] A. Carmona, A 750 GeV graviton from holographic composite dark sectors, Phys. Lett. B 760 (2016) 502 [arXiv: 1603.08913] [INSPIRE].

[60] A. Falkowski and J.F. Kamenik, Diphoton portal to warped gravity, Phys. Rev. D 94 (2016) 015008 [arXiv: 1603.06980] [INSPIRE].

[61] B.M. Dillon and V. Sanz, A little KK graviton at $750 \mathrm{GeV}$, arXiv:1603.09550 [INSPIRE].

[62] J.L. Hewett and T.G. Rizzo, $750 \mathrm{GeV}$ diphoton resonance in warped geometries, arXiv: 1603.08250 [INSPIRE].

[63] A. Kobakhidze, K.L. McDonald, L. Wu and J. Yue, Warped graviton couplings to bulk vectors with brane kinetic terms, arXiv:1606.08565 [INSPIRE].

[64] B.M. Dillon, C. Han, H.M. Lee and M. Park, Confronting diphoton resonance with cascade decays in warped gravity, arXiv:1606.07171 [INSPIRE].

[65] T. Konstandin, G. Nardini and M. Quirós, Gravitational backreaction effects on the holographic phase transition, Phys. Rev. D 82 (2010) 083513 [arXiv:1007.1468] [INSPIRE].

[66] Y. Eshel, S.J. Lee, G. Perez and Y. Soreq, Shining flavor and radion phenomenology in warped extra dimension, JHEP 10 (2011) 015 [arXiv:1106.6218] [INSPIRE].

[67] Z. Chacko, R.K. Mishra and D. Stolarski, Dynamics of a stabilized radion and duality, JHEP 09 (2013) 121 [arXiv:1304.1795] [InSPIRE].

[68] Z. Chacko and R.K. Mishra, Effective theory of a light dilaton, Phys. Rev. D 87 (2013) 115006 [arXiv: 1209.3022] [INSPIRE].

[69] K. Agashe, R. Contino and R. Sundrum, Top compositeness and precision unification, Phys. Rev. Lett. 95 (2005) 171804 [hep-ph/0502222] [INSPIRE].

[70] C. Csáki, J. Hubisz and S.J. Lee, Radion phenomenology in realistic warped space models, Phys. Rev. D 76 (2007) 125015 [arXiv:0705.3844] [INSPIRE].

[71] Z. Chacko, R.K. Mishra, D. Stolarski and C.B. Verhaaren, Interactions of a stabilized radion and duality, Phys. Rev. D 92 (2015) 056004 [arXiv:1411.3758] [INSPIRE].

[72] R. Contino, T. Kramer, M. Son and R. Sundrum, Warped/composite phenomenology simplified, JHEP 05 (2007) 074 [hep-ph/0612180] [INSPIRE].

[73] G. Panico, L. Vecchi and A. Wulzer, Resonant diphoton phenomenology simplified, JHEP 06 (2016) 184 [arXiv: 1603.04248] [INSPIRE].

[74] T. Flacke, B. Gripaios, J. March-Russell and D. Maybury, Warped axions, JHEP 01 (2007) 061 [hep-ph/0611278] [INSPIRE].

[75] C. Vafa and E. Witten, Parity conservation in QCD, Phys. Rev. Lett. 53 (1984) 535 [INSPIRE].

[76] C. Vafa and E. Witten, Restrictions on symmetry breaking in vector-like gauge theories, Nucl. Phys. B 234 (1984) 173 [inSPIRE].

[77] CMS collaboration, Search for resonant production of high mass photon pairs using $12.9 \mathrm{fb}^{-1}$ of proton-proton collisions at $\sqrt{s}=13 \mathrm{TeV}$ and combined interpretation of searches at 8 and $13 \mathrm{TeV}$, CMS-PAS-EXO-16-027 (2016).

[78] ATLAS collaboration, Search for scalar diphoton resonances with $15.4 \mathrm{fb}^{-1}$ of data collected at $\sqrt{s}=13 \mathrm{TeV}$ in 2015 and 2016 with the ATLAS detector, ATLAS-CONF-2016-059 (2016). 
[79] CMS Collaboration, Search for narrow resonances decaying to dijets in pp collisions at $\sqrt{s}=13$ TeV using $12.9 \mathrm{fb}^{-1}$, CMS-PAS-EXO-16-032 (2016).

[80] D. Stolarski and R. Vega-Morales, Directly measuring the tensor structure of the scalar coupling to gauge bosons, Phys. Rev. D 86 (2012) 117504 [arXiv:1208.4840] [INSPIRE].

[81] CMS collaboration, Search for a high-mass resonance decaying into a dilepton final state in $13 \mathrm{fb}^{-1}$ of pp collisions at $\sqrt{\mathrm{s}}=13 \mathrm{TeV}$, CMS-PAS-EXO-16-031 (2016).

[82] ATLAS collaboration, Search for new high-mass resonances in the dilepton final state using proton-proton collisions at $\sqrt{s}=13$ TeV with the ATLAS detector, ATLAS-CONF-2016-045 (2016).

[83] ATLAS collaboration, Search for new resonances decaying to a charged lepton and a neutrino in $p p$ collisions at $\sqrt{s}=13$ TeV with the ATLAS detector, ATLAS-CONF-2016-061 (2016).

[84] CMS collaboration, Search for SSM $W^{\prime}$ production, in the lepton $+M E T$ final state at a center-of-mass energy of 13 TeV, CMS-PAS-EXO-15-006 (2015).

[85] D. Pappadopulo, A. Thamm, R. Torre and A. Wulzer, Heavy vector triplets: bridging theory and data, JHEP 09 (2014) 060 [arXiv: 1402.4431] [INSPIRE].

[86] ATLAS collaboration, Search for diboson resonance production in the $\ell \nu q q$ final state using pp collisions at $\sqrt{s}=13$ TeV with the ATLAS detector at the LHC,

ATLAS-CONF-2016-062 (2016).

[87] ATLAS collaboration, Search for resonances with boson-tagged jets in $15.5 \mathrm{fb}^{-1}$ of $\mathrm{pp}$ collisions at $\sqrt{s}=13$ TeV collected with the ATLAS detector, ATLAS-CONF-2016-055 (2016).

[88] CMS collaboration, Search for resonant $t \bar{t}$ production in proton-proton collisions at $\sqrt{s}=8$ TeV, Phys. Rev. D 93 (2016) 012001 [arXiv:1506.03062] [InSPIRE].

[89] CMS collaboration, Search for $t \bar{t}$ resonances in boosted semileptonic final states in $p p$ collisions at $\sqrt{s}=13$ TeV, CMS-PAS-B2G-15-002 (2015).

[90] R.M. Harris and S. Jain, Cross sections for leptophobic topcolor $Z^{\prime}$ decaying to top-antitop, Eur. Phys. J. C 72 (2012) 2072 [arXiv:1112.4928] [InSPIRE].

[91] K. Agashe, A. Belyaev, T. Krupovnickas, G. Perez and J. Virzi, LHC signals from warped extra dimensions, Phys. Rev. D 77 (2008) 015003 [hep-ph/0612015] [INSPIRE].

[92] R.S. Chivukula, A. Farzinnia, J. Ren and E.H. Simmons, Hadron collider production of massive color-octet vector bosons at next-to-leading order, Phys. Rev. D 87 (2013) 094011 [arXiv:1303.1120] [INSPIRE].

[93] H. Georgi and Y. Nakai, Diphoton resonance from a new strong force, Phys. Rev. D 94 (2016) 075005 [arXiv: 1606.05865] [INSPIRE].

[94] Z. Chacko, H.-S. Goh and R. Harnik, The twin Higgs: natural electroweak breaking from mirror symmetry, Phys. Rev. Lett. 96 (2006) 231802 [hep-ph/0506256] [INSPIRE].

[95] A. Arvanitaki, N. Craig, S. Dimopoulos and G. Villadoro, Mini-split, JHEP 02 (2013) 126 [arXiv: 1210.0555] [INSPIRE].

[96] N. Arkani-Hamed, A. Gupta, D.E. Kaplan, N. Weiner and T. Zorawski, Simply unnatural supersymmetry, arXiv: 1212.6971 [INSPIRE]. 
[97] I.I. Kogan, S. Mouslopoulos, A. Papazoglou and L. Pilo, Radion in multibrane world, Nucl. Phys. B 625 (2002) 179 [hep-th/0105255] [INSPIRE].

[98] R. Sundrum, Effective field theory for a three-brane universe, Phys. Rev. D 59 (1999) 085009 [hep-ph/9805471] [INSPIRE].

[99] Z. Chacko, M.A. Luty and E. Ponton, Massive higher dimensional gauge fields as messengers of supersymmetry breaking, JHEP 07 (2000) 036 [hep-ph/9909248] [INSPIRE].

[100] H. Davoudiasl, G. Perez and A. Soni, The little Randall-Sundrum model at the Large Hadron Collider, Phys. Lett. B 665 (2008) 67 [arXiv:0802.0203] [INSPIRE]. 